\title{
Aerodynamic Shape Optimization of Highly Nonplanar Raised and Drooped Wings
}

\author{
Conlan-Smith, Cian; Ramos-García, Néstor; Schousboe Andreasen, Casper
}

Published in:

Journal of Aircraft

Link to article, DOI:

10.2514/1.C036403

Publication date:

2022

Document Version

Peer reviewed version

Link back to DTU Orbit

Citation (APA):

Conlan-Smith, C., Ramos-García, N., \& Schousboe Andreasen, C. (2022). Aerodynamic Shape Optimization of Highly Nonplanar Raised and Drooped Wings. Journal of Aircraft, 59(1), 206-218.

https://doi.org/10.2514/1.C036403

\section{General rights}

Copyright and moral rights for the publications made accessible in the public portal are retained by the authors and/or other copyright owners and it is a condition of accessing publications that users recognise and abide by the legal requirements associated with these rights.

- Users may download and print one copy of any publication from the public portal for the purpose of private study or research.

- You may not further distribute the material or use it for any profit-making activity or commercial gain

- You may freely distribute the URL identifying the publication in the public portal

If you believe that this document breaches copyright please contact us providing details, and we will remove access to the work immediately and investigate your claim. 


\title{
Aerodynamic shape optimization of highly non-planar raised and drooped wings
}

\author{
Cian Conlan-Smith *, Néstor Ramos-García ${ }^{\dagger}$ and Casper Schousboe Andreasen ${ }^{\ddagger}$ \\ Technical University of Denmark, DK-2800 Lyngby, Denmark.
}

\begin{abstract}
Aircraft wings are commonly designed with non-planar geometry, such as winglets, in order to improve aerodynamic efficiency. This work presents a method for generating nonplanar wing designs through gradient-based optimization, which is then used to investigate the performance characteristics of non-planar wings. The non-planar parameterization is defined to give a large design space that allows the formation of highly non-planar features and permits large changes to the geometry. Aerodynamic characteristics are captured using an inviscid 3D panel method with approximations for viscous drag. The methodology is demonstrated by optimizing reference wings from literature and comparing aerodynamic performance. Investigations are also performed on the impact on performance when wings are raised or drooped, and the differences in aerodynamic behavior between the two designs. Results suggest that the converged designs and their performance is highly dependent on how the geometry is restricted. If a large design space is provided, both raised and drooped wings are able to produce designs with similar performance when only inviscid analysis is considered. When accounting for viscous effects, results suggest that drooped wings are not beneficial for drag reduction.
\end{abstract}

\section{Nomenclature}

A $=$ doublet aerodynamic influence coefficient matrix

$a=x$-coordinate of quarter chord curve

B = source aerodynamic influence coefficient matrix

$b \quad=$ wingspan

$C_{d}=\frac{d}{\frac{1}{2} \rho_{\infty} V_{\infty}^{2} c}$, sectional drag coefficient

$C_{D}=\frac{D}{\frac{1}{2} \rho_{\infty} V_{\infty}^{2} S}$, drag coefficient

$C_{D_{i}}=\frac{D_{i}}{\frac{1}{2} \rho_{\infty} V_{\infty}^{2} S}$, induced drag coefficient

\footnotetext{
* Postdoctoral researcher, Department of Mechanical Engineering, Section of Solid Mechanics, cicosm@mek.dtu.dk. Member AIAA

${ }^{\dagger}$ Senior Researcher, Department of Wind Energy, Section of Aero and Fluid Dynamics.

$\doteqdot$ Associate Professor, Department of Mechanical Engineering, Section of Solid Mechanics.
} 


$$
\begin{aligned}
& C_{l}=\frac{l}{\frac{1}{2} \rho_{\infty} V_{\infty}^{2} c} \text {, sectional lift coefficient } \\
& C_{L}=\frac{L}{\frac{1}{2} \rho_{\infty} V_{\infty}^{2} S} \text {, lift coefficient } \\
& C_{P}=\text { pressure coefficient } \\
& c=\text { chord length } \\
& d \quad=\text { sectional drag } \\
& D=\text { drag } \\
& D_{i}=\text { induced/inviscid drag } \\
& D_{v} \quad=\text { viscous drag } \\
& e \quad=\text { efficiency factor } \\
& L \quad=\text { lift } \\
& n=\text { normal vector } \\
& q \quad=\quad z \text {-coordinate of quarter chord curve } \\
& S=\text { wing planform area } \\
& V \quad=\text { velocity } \\
& \mathbf{x}=\text { design variables } \\
& \alpha=\text { angle of attack } \\
& \alpha_{i} \quad=\quad \text { induced angle of attack } \\
& \eta \quad=\quad \frac{2 y}{b} \text {, normalized span location } \\
& \mu \quad=\text { doublet strength } \\
& \rho \quad=\text { density } \\
& \sigma \quad=\quad \text { source strength } \\
& \mathbb{R}=\frac{b^{2}}{S} \text {, aspect ratio }
\end{aligned}
$$

Subscripts

$t \quad=$ associated with wingtip

$\infty \quad=$ associated with freestream

\section{Introduction}

Studies on optimal designs for aircraft wings date back to at least the 1920s where Munk [1] derived that an elliptically loaded planar wing is required to achieve a minimum induced drag. This has since become common knowledge where an elliptic planform wing is the optimal design for planar, untwisted, unswept wings. When considering non-planar and swept wings there is no definitive optimal design, however certain non-planar wings have been shown to improve efficiencies beyond that of the elliptic wing. The most notable early study in this area comes from Cone [2] who derived 
a mathematical basis for achieving an optimal span loading for non-planar wings in order to minimize induced drag.

Optimization methods are frequently used to generate wing designs that achieve optimal performance with respect to a given set of conditions, e.g. fixed lift and span, or improve upon the performance of a reference design. There are multiple methods in which this can be implemented where methods generally differ in their computational expense and fidelity. Cone's method [2] (introduced previously), is one such example to achieve optimal span loading. Demasi [3] developed a similar approach to Cone, which was based on lifting lines to achieve optimal span loading for fixed lift and was later extended to closed wing systems [4] and non-planar wings [5]. These low-fidelity methods allow fast calculations where optimized designs can be achieved in a relatively short time frame. However, the resulting designs only achieve spanwise loading and the non-planar wing shape. Using higher fidelity methods can achieve optimal span loading indirectly through shape optimization where local geometry such as chord and twist are allowed to vary. Some examples of these methods include Hicken et al [6] and Gagnon et al [7] who solve aerodynamic optimization problems based on the Euler equations. Secco and Martins [8] conducted similar optimization studies on truss braced wings by solving the Reynolds averaged Navier-Stokes (RANS) equations which account for viscous effects thus offering a further increase in fidelity. However, the computational cost is greatly increased as the fidelity of the model increases. Panel methods [9, 10] offer a good trade-off between fidelity and computational cost, and have previously been applied to aerodynamic and aeroelastic shape optimization in [11, 12]. This difference in computational cost is further increased when large changes in geometry is permitted throughout the optimization routine as finite volume based methods used to solve Euler or RANS equations can require regular remeshing to prevent mesh distorsion. This large overhead is avoided with panel methods as they only require a surface mesh making it easier to handle large changes in geometry.

Recent years have seen a growing interest in bio-inspired wing design, in order to achieve designs that are believed to be close to optimal. The motivation is that birds have naturally evolved over the course of millions years to develop highly efficient wings. Van Dam [13], inspired by the caudal fins of fish, studied the performance of crescent shaped wings and found that the aerodynamic efficiency of an elliptic wing can be improved by introducing a backward curved sweep. Lazos [14] compared different bio-inspired wing configurations to the elliptic wing to demonstrate the possible gains in efficiency. The most noteworthy configuration is a hyper elliptic cambered span (HECS) drooped wing, which was inspired by the shape of seagull wings in high-speed gliding flight, and produced a large lift-to-drag ratio over a wide range of $C_{L}$ values. The concept of a drooped wing is represented in Fig. 11 where the wing's spanwise camber is downward (or drooped) compared to more traditional wings which may be raised to create features such as a winglet. Lazos and Visser [15] later compared different HECS wing designs with conventional wing configurations (such as a tapered wing with winglet) which also demonstrated large gains in lift-to-drag ratios.

Nguyen et al [16] conducted parameter studies on the aeroelastic performance of aircraft wing concepts, where the authors present large reductions in drag when drooped wings are utilized. The drooped wing designs here are again motivated by seagull wings in gliding flight. Khosravi and Zingg [17] conducted aeroelastic optimization studies of 


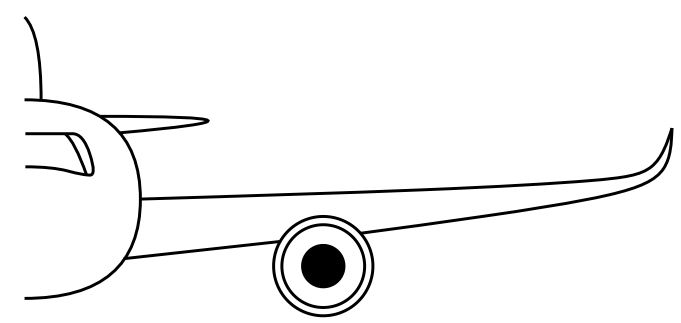

(a)

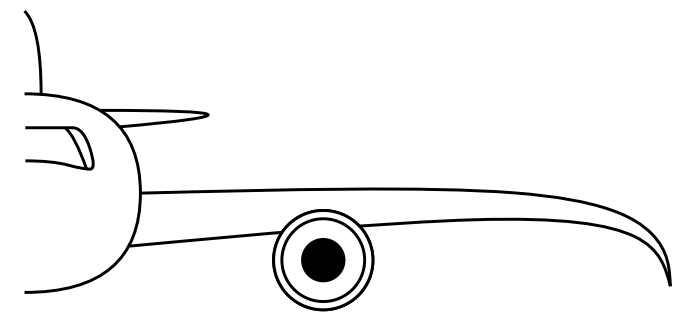

(b)

Fig. 1 Concept for (a) raised and (b) drooped wings.

drooped wings which produce efficiency gains of up to $5 \%$ compared to optimized planar wings.

There are also some conflicting studies that did not predict increased performance for drooped wings. Ranjan et al [18] performed drag minimization studies on both raised and drooped HECS wings with tapered unswept planforms. The optimization procedure was based on Cone's method of optimal span loading which predicted the same performance for raised and drooped HECS wings. Solving the RANS equations post-optimization showed that raised wings outperformed drooped, but both improved on the elliptic wing. Liersh et al [19], and Pomeroy and Visser [20] both present studies on spanwise cambered elliptic wings and found that reductions in drag was achieved with raised wings, but not with drooped wings. However, the wings were drooped to a much lesser extent than those in previously mentioned studies, with inclinations of up to 15 degs and 30 degs respectively, versus almost 90 degs.

There is currently no agreement in the literature on whether drooped wings offer a more efficient alternative to more conventional raised winglet designs, with different studies drawing conflicting conclusions. However, there are few examples in literature where these comparisons are based on designs achieved through numerical optimization procedures. Of those examples that have treated this as an optimization problem, most have done so with a relatively restricted design space. The current work applies gradient-based optimization routines to design highly non-planar wings whose geometry is defined on a spanwise sectional basis, thus creating a large design space. Subsequently the method is used to investigate the potential gains for optimizing non-planar wings compared to planar and non-planar designs presented in literature. Further optimization problems are presented with both raised and drooped wings, their optimized designs compared in terms of performance, and additional studies are include explain these differences and aid discussion.

In the current work the methodology in [11] is extended to include non-planar wing parameterizations and a novel 
method of including viscous drag approximations in panel methods for non-planar wings. The following section will discuss the non-planar parametrization and discretization of the model. Panel methods are summarized in Section III] including lift and drag calculation methods. The results section is subdivided into 3 subsections detailing a comparison of optimized designs to those in the literature, an investigation on raised vs. drooped wings, and a study accounting for viscous effects. Finally, the findings are concluded in Section[V Additionally, 2 appendices are included, Appendix A studies the effect of mesh discretization on results, and B presents a comparison between numerical results from the current model and experimental results from literature.

\section{Discretization \& parameterization}

The design parameterization and panel discretization of the wing is shown in Fig. 2. A series of airfoil sections are assembled on a discrete quarter chord curve that is defined by control points on the curve with $x$-coordinates, $\boldsymbol{a}$, and $z$-coordinates, $\boldsymbol{q}$. The airfoil sections are defined within a plane that is normal to the quarter chord curve from the perspective of the $x$-axis. Within this plane, airfoils have a twist angle, $\theta$, measured from the $x$-axis. The control points on the quarter chord curve are defined with a half cosine distribution in the span-wise direction, quadrilateral panels are defined between each set of neighboring airfoil sections, and all problems are solved with a mesh size of 40x100 (refer to Appendix $\mathrm{A}$ for impact of mesh size), i.e. 41 airfoil sections are defined.

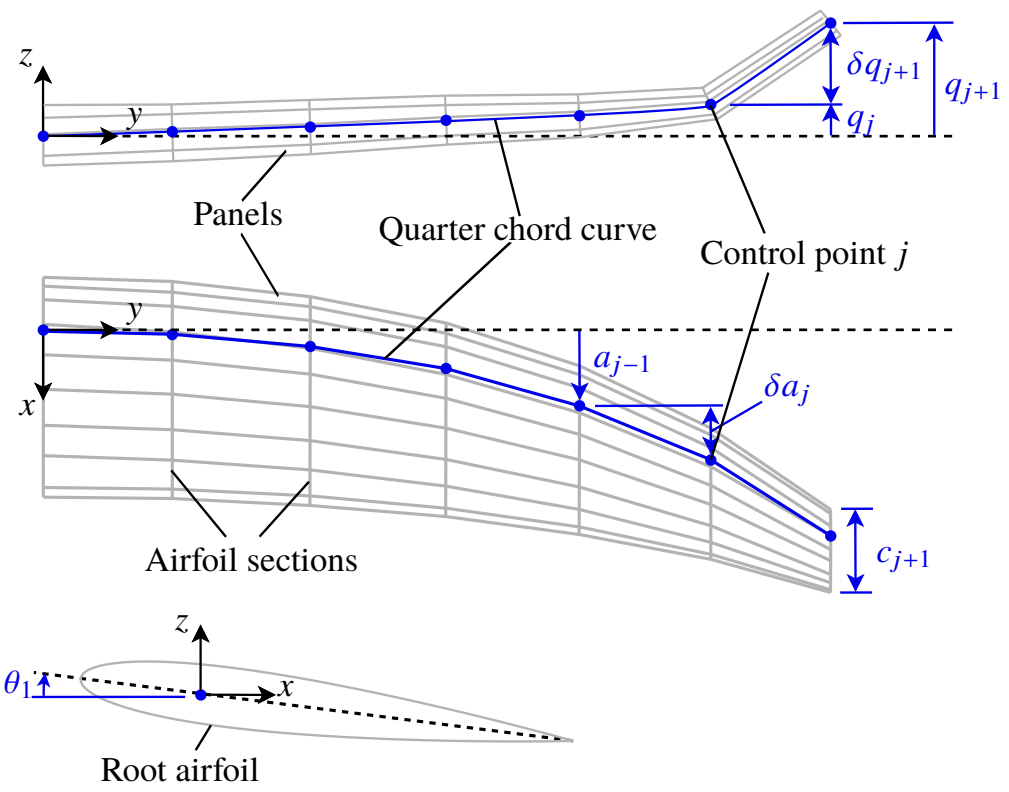

Fig. 2 Parametrization of the wing geometry with elevation (top), planform (middle), and root airfoil (below). A quarter-chord curve is defined by parameters $a$ and $q$, where airfoils are assembled normal to the curve from the perspective of the $x$-axis.

The sectional parameters $\boldsymbol{c}, \boldsymbol{a}, \boldsymbol{q}$ and $\boldsymbol{\theta}$, shown in Fig. 2, can be controlled using design variables, $\mathbf{x}$. Design variables are used to define $\boldsymbol{\theta}$ explicitly at each section, but all other design variables define the change in the parameter, 
e.g. $\delta \boldsymbol{q}$, where $q_{j+1}=q_{j}+\delta q_{j+1}$. By defining the design variables in this manner it is easy to enforce that parameters are monotonically increasing/decreasing along the span, avoiding fluctuating geometries and requiring less regularization.

To ensure smooth designs and avoid numerical artefacts the design variables are filtered with neighboring sections in the spanwise direction as described in [11]. Previous work has demonstrated that when an inviscid model is used only small improvements can be gained by optimizing airfoil sections along the wing [11]. In the current work, the focus is on investigating the potential gains of non-planar geometry, and as such all examples will use constant NACA0012 airfoil profiles throughout the span. However, all methods can easily be extended to allow varying cross sections [11, 12].

\section{Panel Methods}

The aerodynamic characteristics are captured using a 3D panel method introduced in previous works [11, 12, 21]. A Dirichlet boundary condition is implemented using constant source-doublet panels for the wing, and constant doublet panels in the wake. The governing equation for the panel method is defined as

$$
\sum_{k=1}^{N_{p}}\left[a_{j, k}(\mathbf{x}) \mu_{k}+b_{j, k}(\mathbf{x}) \sigma_{k}(\mathbf{x})\right]=0
$$

where $N_{p}$ is the number of panels, $\mu_{k}$ and $\sigma_{k}$ are the doublet and source strengths of panel $k$, and $a_{j, k}$ and $b_{j, k}$ are the aerodynamic influence coefficients (AIC) for calculating the potential at the collocation point in the center of panel $j$ due to the doublet and source of panel $k$. Equation 1 is then enforced at each of the $N_{p}$ collocation points resulting in a system of equations of size $N_{p} \times N_{p}$. Derivations of AIC's are provided in [22], and using a zero-internal perturbation formulation yields $\sigma_{k}=\boldsymbol{V}_{\infty} \cdot \mathbf{n}_{k}$, where $\mathbf{n}_{k}$ is the normal vector of panel $k$. The doublet strength $\boldsymbol{\mu}$, can then be solved for using (1).

Once the doublet distribution is known, $\boldsymbol{\mu}$ can be differentiated numerically over the surface of the wing to yield the velocity, $\boldsymbol{V}_{k}$, at panel $k$ 's center. Given the surface velocities, the coefficient of pressure can be calculated via the Bernoulli equation.

$$
C_{P, k}=1-\frac{\left\|V_{k}\right\|^{2}}{V_{\infty}^{2}}
$$

Lift and drag could be calculated from $C_{P}$ distributions, however, surface pressure integration is prone to numerical errors which can create non-physical results for unconventional wing designs where there is a large change in panel geometry. As the optimizer is likely to exploit these numerical weaknesses, a Trefftz integration method is used which has proven to be less susceptible to numerical errors [11, 23]. Using Trefftz plane integration, the lift and induced drag can be calculated as

$$
D_{i}=-\frac{1}{2} \rho_{\infty} \sum_{j=1}^{N_{s}} \mu_{j} s_{j} v_{j} \cdot \boldsymbol{n}_{j}
$$




$$
L=\rho_{\infty} V_{\infty} \sum_{j=1}^{N_{s}} \mu_{i} \cos \left(\beta_{j}\right) s_{j}
$$

where $s_{j}, \beta_{j}$, and $\boldsymbol{n}_{j}$ are the length, orientation, and normal vector of the wake panel in the Trefftz plane at section $j$, and $N_{s}$ is the number of panels in the spanwise direction. The wake geometry is described by projecting the trailing edge downstream in the freestream direction, and extends 30 semispans downstream. Velocities in the Trefftz plane, $v_{j}$, are calculated using the circulation around the wake panel edge, $\Gamma_{k}$ as defined in [24]

$$
\boldsymbol{v}_{j}=\frac{1}{2 \pi} \sum_{k=1}^{N_{s}} K_{k} \Gamma_{k} \frac{\hat{\boldsymbol{x}} \times \mathbf{r}_{j k}}{\left\|\mathbf{r}_{j k}\right\|^{2}}
$$

where $\hat{\boldsymbol{x}}$ is the normal vector of the Trefftz plane, $\mathbf{r}_{j k}$ is a spatial vector between the wake panel edge and the calculation point $j$, and $K_{k}$ is a kernel which desingularizes the vortex core and is defined using the definition in [25] as

$$
K=\frac{\left\|\mathbf{r}_{j k}\right\|^{2}}{\left(r_{c}^{2 \zeta}+\left\|\mathbf{r}_{j k}\right\|^{2 \zeta}\right)^{\frac{1}{\zeta}}}
$$

where $r_{c}$ is the viscous core radius which we define as $20 \%$ of the chord length, and $\zeta$ is taken to be 2 which represents a Lamb-Oseen vortex model. It may be tempting to neglect the core model (i.e. $K=1$ ), however this can lead to large numerical errors with fine panel meshes as the calculation point comes very close to the vortex center.

The total drag is then computed as the sum of induced and viscous drag, i.e. $D=D_{i}+D_{v}$, where viscous drag can be calculated as

$$
D_{v}=\frac{1}{2} \rho_{\infty} V_{\infty}^{2} \sum_{j=1}^{N_{s}} c_{j} s_{j} C_{d, j}
$$

where $s_{j}$ is the length of the wing section $j$, and $C_{d, j}$ the local drag coefficient. $C_{d}$ is calculated from $2 \mathrm{D}$ airfoil data (generated via XFoil [26]) by interpolating with respect to the local effective angle of attack, $\alpha_{e f}$, and Reynolds number. It is important to note that as $C_{d}$ is calculated using 2D airfoil data, spanwise flow is not considered in the viscous drag calculation.

Airfoils are defined in the center of each wing section within in a plane normal to the quarter chord line as shown in Fig. 3 . The Reynolds number is calculated using the freestream velocity within the airfoil plane and the chord length at each wing section. A method is introduced for calculating the effective angle of attack which accounts for non-planar geometry, twist, freestream angle of attack, and induced angle of attack. The effective angle of attack is measured within the airfoil plane shown in Fig. 3, where a reference system, $(\hat{x}, \hat{z})$, is defined within the plane such that $\hat{x}$ is parallel with the global $x$-axis. The freestream velocity within the plane is then

$$
\hat{\boldsymbol{V}}_{\infty}=\left\{V_{\infty, x}, V_{\infty, z} \cos (\hat{\psi})-V_{\infty, y} \sin (\hat{\psi})\right\}^{\mathrm{T}}
$$




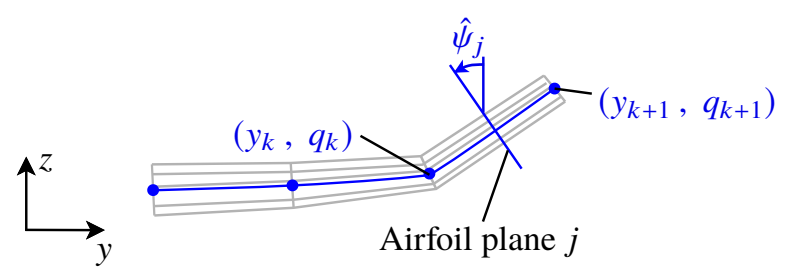

(a) $y-z$ plane

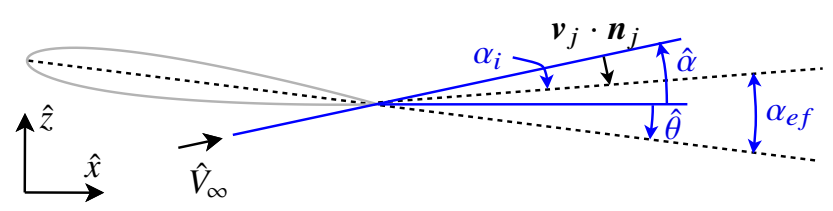

(b) Airfoil plane $j$

Fig. 3 Schematic indicating angles for effective angle of attack calculation: (a) definition of airfoil plane, and (b) airfoil section with relevant angles.

where $\hat{\psi}$ is the inclination of the wing section as represented in Fig. 3 3 . sin and cos terms can be calculated from the control points on the quarter chord curve as

$$
\sin \left(\hat{\psi}_{j}\right)=\frac{q_{k+1}-q_{k}}{\sqrt{\left(\left(y_{k+1}-y_{k}\right)^{2}+\left(q_{k+1}-q_{k}\right)^{2}\right)}} \quad, \quad \cos \left(\hat{\psi}_{j}\right)=\frac{y_{k+1}-y_{k}}{\sqrt{\left(\left(y_{k+1}-y_{k}\right)^{2}+\left(q_{k+1}-q_{k}\right)^{2}\right)}}
$$

The effective angle of attack can be calculated as the sum of angles shown in Fig. $3 \mathrm{p}$

$$
\alpha_{e f}=\hat{\alpha}+\hat{\theta}-\alpha_{i}
$$

where $\hat{\alpha}$ is angle of attack within the plane which can be calculated from $\hat{\boldsymbol{V}}, \hat{\theta}$ is the twist of the wing section, and $\alpha_{i}$ is the induced angle of attack. The induced angle of attack is calculated using the downwash velocity in the Trefftz plane, $v_{j}$

$$
\left\{\alpha_{i}\right\}_{j}=\tan ^{-1}\left(\frac{\boldsymbol{v}_{j} \cdot \boldsymbol{n}_{j}}{\left\|\hat{\boldsymbol{V}}_{\infty}\right\|}\right) \simeq \frac{\boldsymbol{v}_{j} \cdot \boldsymbol{n}_{j}}{\left\|\hat{\boldsymbol{V}}_{\infty}\right\|}
$$

where $\alpha_{i}$ is in-plane with the airfoil section. Note that $\hat{\theta}$ is associated with the wing section, as opposed to $\theta$, in Fig. 2 , which represent values at the control points.

\section{Results}

In this section, optimization procedures will be used to maximize the aerodynamic performance of the wing. One important parameter for comparing the performance of aircraft wings is the span efficiency factor defined as

$$
e=\frac{C_{L}^{2}}{\pi R C_{D_{i}}}
$$


where $\not R$ is the wing's aspect ratio, and only the induced drag is considered. The parameter, $e$, compares the efficiency of a wing to that of an elliptic wing which has a value $e=1$. The efficiency is constant for a given wing irrespective of angle of attack or freestream velocity (for incompressible inviscid flow). An alternative parameter for characterizing performance is the lift-to-drag ratio, ${ }^{L} / D$, where typically the viscous drag is also included. Unlike $e,{ }^{L} / D$ varies with angle of attack and freestream velocity. When viscous drag is not included, $L / D$ is unrealistically high for low angles of attack and singular for zero lift as there will be no induced drag. If viscous drag is included, ${ }^{L} / D$ will be small for low and high angles of attack, and have an optimum $\alpha$ where ${ }^{L} / D$ is at a maximum.

Optimization studies are solved using the gradient-based method of moving asymptotes [27], where the initial design is a rectangular untwisted planar wing with all constraints satisfied. All sensitivity analysis is conducted using a discrete adjoint approach where gradient derivations can be found in [11]. The optimization problem is terminated once the relative change in design variables and the objective function between iterations is less than $\epsilon=2 \times 10^{-3}$, and all constraints are satisfied to within $\epsilon$.

\section{A. A Comparison with Reference Designs}

Consider the optimization problem defined in 13 , where the objective is to maximize the efficiency factor, $e$, with a fixed aspect ratio, $R$, and constraints on $a$ and $q$ values at the wingtip (notated by subscript $t$ ).

$$
\begin{array}{ll}
\min _{\mathbf{x} \in \mathbb{R}^{N_{\mathrm{x}}}} & :-e(\mathbf{x}, \boldsymbol{\mu}) \\
\text { subject to } & : \mathbb{R}(\mathbf{x})=\mathbb{R}_{0} \\
& \left|a_{t}(\mathbf{x})\right| \leq \bar{a}_{t} \\
& \left|q_{t}(\mathbf{x})\right| \leq \bar{q}_{t} \\
& \underline{\mathrm{x}} \leq \mathrm{x}_{j} \leq \overline{\mathrm{x}} \quad \text { for } j=1, \ldots, N_{\mathrm{x}}
\end{array}
$$

Optimized designs are compared to three reference cases from the literature where each wing has the same aspect ratio, $\mathbb{R}_{0}=7$. The three reference wings are shown in Fig. 4 A-c. The first case is has a standard elliptic planform which is the known optimal design for planar, unswept, untwisted wings. The second is a planar crescent wing introduced in [13], which was motivated by caudal fins of fish and has an elliptic chord and sweep distribution. The final reference case is a non-planar wing with a hyper-elliptic cambered span (HECS). The HECS wing was introduced in [14] and was motivated by the shape of seagull wings in gliding flight. The original HECS wing was comprised of SD7032 airfoils, however for consistency with other designs NACA 0012 airfoils are used here instead. By allowing specific design variables we can create appropriate comparisons to these reference wings. An elliptic wing will correspond to solving (13) with only chord length defined by design variables, i.e. $\mathbf{x}=\delta \mathbf{c}$. Likewise the crescent and HECS wings can be compared to designs achieved with $\mathbf{x}=\{\delta \mathbf{c}, \delta \boldsymbol{a}\}$ and $\mathbf{x}=\{\delta \mathbf{c}, \delta \boldsymbol{a}, \delta \boldsymbol{q}\}$, respectively. Optimized designs are shown in 
Fig. $4 \mathrm{~d}-\mathrm{f}$, and the efficiency of reference and optimized designs are shown in Table 1 .

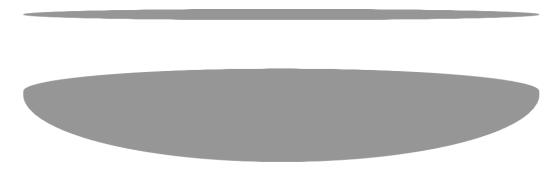

(a) Elliptic

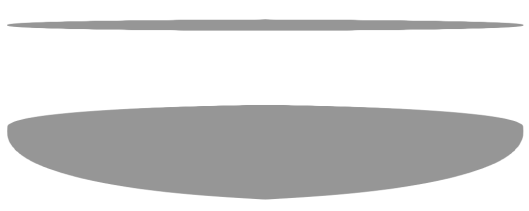

(d) $\mathbf{x}=\delta \mathbf{c}$

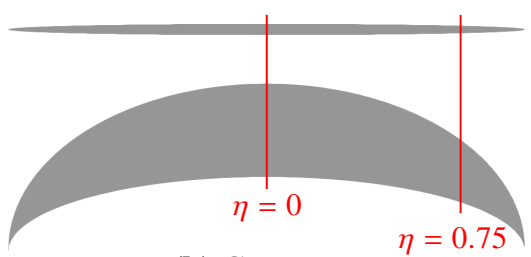

(b) Crescent

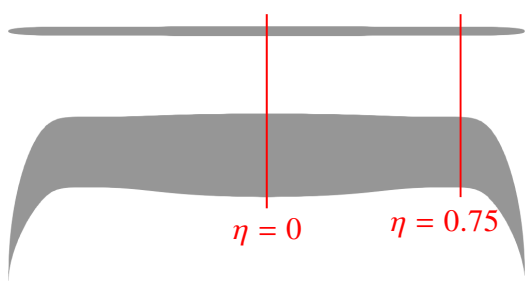

(e) $\mathbf{x}=\{\delta \mathbf{c}, \delta \boldsymbol{a}\}$

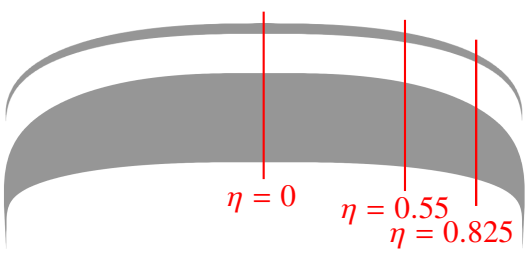

(c) HECS

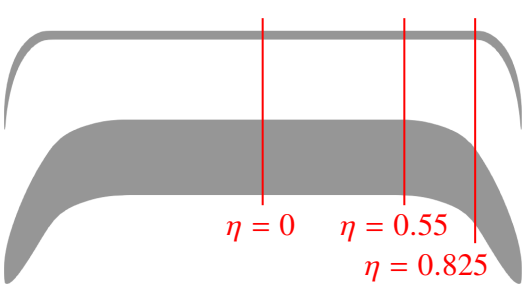

(f) $\mathbf{x}=\{\delta \mathbf{c}, \delta \boldsymbol{a}, \delta \boldsymbol{q}\}$

Fig. 4 Elevation and planform views for reference designs, (a)-(c), and optimized designs, (d)-(f). Markers are included at $\eta$ locations when wings begin to sweep and droop ( $\eta=0$ for reference wings).

\begin{tabular}{lclc}
\hline \hline Reference & $e_{\text {ref }}$ & Optimized & $e_{\text {opt }}$ \\
\hline Elliptic & 1.004 & $\mathbf{x}=\delta \mathbf{c}$ & 1.004 \\
Crescent & 1.019 & $\mathbf{x}=\{\delta \mathbf{c}, \delta \boldsymbol{a}\}$ & 1.027 \\
HECS & 1.168 & $\mathbf{x}=\{\delta \mathbf{c}, \delta \boldsymbol{a}, \delta \boldsymbol{q}\}$ & 1.291 \\
\hline \hline
\end{tabular}

Table 1 Comparison of efficiency factors for reference wings and optimized designs defined by design variables, x. All designs have an aspect ratio of 7 .

An elliptic wing is known that to have an efficiency, $e=1$, which is closely predicted by the model with only a $0.4 \%$ error. The optimized design for $\mathbf{x}=\delta \mathbf{c}$ converges to the same elliptic wing and hence the same performance is achieved. As this is the known optimal design, this case helps to validate the optimization procedure.

The crescent wing predicts an increase in efficiency of $1.5 \%$ over the elliptic wing. When the crescent wing was first introduced in [13] an increase in efficiency of $8 \%$ was predicted. The difference in $e$ is likely due to differences in discretization or drag calculation methods. For example $e$-factors in Table 1 are achieved with Trefftz plane method, but calculating lift and drag directly from pressure integration yields values of $e=1.085$, giving a $8.5 \%$ increase. Van Dam later conducted experimental investigations into crescent wings and found increases in efficiency of between 2 and $4 \%$ [28]. As previously stated, the Trefftz method is used because it less susceptible to numerical error such as discretization errors. Smith [23] demonstrates that $e$-factors from $C_{P}$ integration are very the dependent on the mesh, whereas Trefftz plane calculations are not. When setting $\mathbf{x}=\{\delta \mathbf{c}, \delta \boldsymbol{a}\}$, the optimizer converges to a very different planform than that of the crescent wing, and with increase in $e$ of $2.3 \%$. Rather than gradually sweeping the wing, the optimized design is straight until $\eta \simeq 0.75$ before drastically sweeping to create raked wing tips. 
The HECS wing produces an increase of $16.4 \%$ in efficiency compared to the elliptic wing, whereas the optimized design for $\mathbf{x}=\{\delta \mathbf{c}, \delta \boldsymbol{a}, \delta \boldsymbol{q}\}$ produces $28.7 \%$ increase. The optimized design is similar to the HECS wing in that it has produced swept drooped wings. However, the location in which the wings start to sweep and droop are further outboard than the HECS wing, which has a gradual change in sweep and droop from $\eta=0$. The optimized wing starts to sweep around $\eta \simeq 0.55$, maintains a constant chord until this point, and droops around $\eta \simeq 0.825$. Both constraints on $a_{t}$ and $q_{t}$ are active for this case, which indicates that larger $e$-factors can be achieved if the constraints were relaxed. As mentioned previously, the airfoil has been modified from the original paper. When optimized with the original SD7032 airfoils, the optimization problem converges to a similar designs to that shown in Figure 1 f. A similar difference is also seen between reference and optimized designs with SD7032 airfoils where $e$ is 1.165 and 1.293, respectively.

We further investigate the location of sweep and droop by taking a closer look at the different HECS wing designs. The geometry of HECS wings are defined by the equation for a hyper ellipse. Using the wing parametrization in Fig. 2 . equivalent HECS wing geometries can be achieved through defining hyper elliptic distributions of $c, a$, and $q$ as

$$
\begin{aligned}
& \left(\frac{c}{c_{\max }}\right)^{p}+\left(\frac{y}{0.5 b}\right)^{p}=1 \\
& \left(\frac{a_{t}-a}{a_{t}}\right)^{p}+\left(\frac{y}{0.5 b}\right)^{p}=1 \\
& \left(\frac{q_{t}-q}{q_{t}}\right)^{p}+\left(\frac{y}{0.5 b}\right)^{p}=1
\end{aligned}
$$

where for a hyper elliptic distribution $p>2$, and $p=2$ corresponds to an elliptic distribution. The reference HECS wing used $p=2.5$, but with a slightly different definition where leading and trailing edges were defined rather than $c$ and $a$ distributions, refer to [14] for definition. Each equation is defined with the same $p$-value where $q_{t}$ and $a_{t}$ values are defined to be equal to that of the HECS reference wing, and $q_{t}$ is negative for drooped wings. The aspect ratio is kept constant which corresponds to the following definition for maximum chord length (at the root)

$$
c_{\text {max }}=\frac{b}{\mathscr{R}} \frac{\mathcal{F}\left(\frac{p+2}{p}\right)}{\mathcal{F}\left(\frac{p+1}{p}\right)^{2}}
$$

where the operator $\mathcal{F}(\square)$ represents the gamma function, which is defined for positive real numbers as

$$
\mathcal{F}(u)=\int_{0}^{\infty} \exp (-t) t^{u-1} d t \quad \text { where } u \in \mathbb{R}, u>0
$$

Fig. 5 shows the change in $e$-factors for HECS wings produced with different values for $p$. By increasing the value of $p$ the wing remains approximately planar for a larger portion of the inboard span and the spanwise location where the 
wing begins to droop is pushed further outboard creating a larger curvature in the cambered span. From Fig. 5 a, this HECS parameterization has an optimum $p$-value where $e$ is maximum at approximately $p=6$. Referring to Figs. 55 and 5 , the design with $p=6$ has a similar spanwise camber to that of the optimized design. However, the optimized design has predicted a greater efficiency due to the freedom to deviate from a hyper elliptic distribution, and create different sweep and chord distributions.

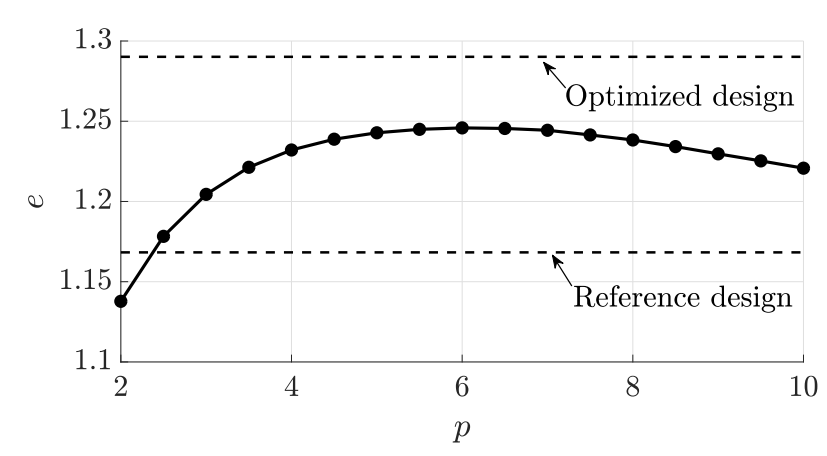

(a)

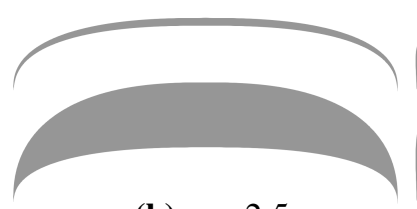

(b) $p=2.5$

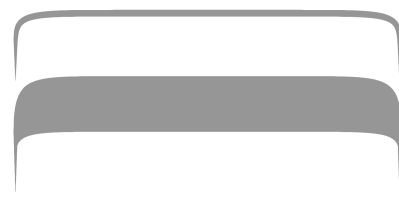

(d) $p=10$

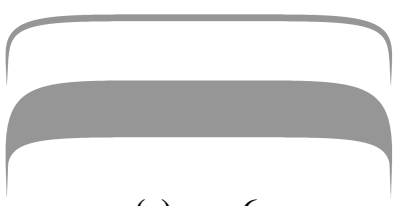

(c) $p=6$

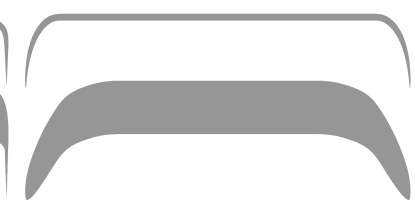

(e) Optimized Design

Fig. 5 (a) Variation in efficiency, $e$, with respect to the exponent, $p$, used in the HECS wing definition given in [14). $e$-factors also shown for reference and optimized designs shown in Figs $4 \mathrm{c}$ and $4 \mathrm{f}$ respectively. (b-d) Wing geometries for different values of $p$, and (e) Optimized wing geometry

\section{B. Raised vs. Drooped Wings}

The optimized results shown in Fig. 4 demonstrate that large increases in efficiency can be seen achieved when $q$-values are allowed to change. But the previous study only permitted drooped wings to form in order to create a comparison with the reference HECS wing. The following studies aim to address the question of whether negative $q$-values (drooped wings) can achieve a higher efficiency than positive $q$-values (raised wings), and how the resulting designs achieve gains in efficiency compared to planar wings.

Consider the optimization problem in (17), with the objective to maximize $e$, and an upper constraint on the wingtip value of $q$. Here, only one design variable per section is included, $\mathbf{x}=\delta \boldsymbol{q}$, where $\boldsymbol{a}=\mathbf{0}$, and $\boldsymbol{c}=b / \boldsymbol{R}_{0}$ to give a rectangular planform with an aspect ratio equal to that of previous studies.

$$
\begin{array}{ll}
\min _{\mathbf{x} \in \mathbb{R}^{N_{\mathrm{x}}}} & :-e(\mathbf{x}, \boldsymbol{\mu}) \\
\text { subject to } & :\left|q_{t}(\mathbf{x})\right| \leq \bar{q}_{t} \\
& \underline{\mathrm{x}} \leq \mathrm{x}_{j} \leq \overline{\mathrm{x}} \quad \text { for } j=1, \ldots, N_{\mathrm{x}}
\end{array}
$$

Optimization results are shown in Fig. 6 for $q>0$ and $q<0$, and are compared to a planar wing of equal planform. Spanwise lift and drag distributions, as well as $C_{p}$ distributions at specific cross sections are shown in Fig. 7 for an angle of attack of $3.5 \mathrm{deg}$, and efficiency factors for each design are given in Table 2 


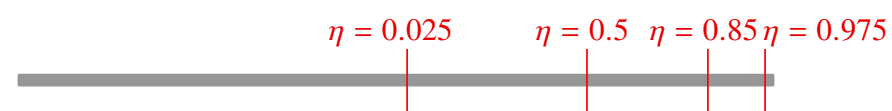

(a) Planar, $q=0$

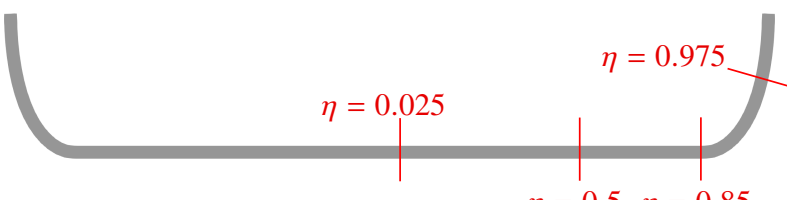

(b) Rasied, $q>0$ i=0.5 $\eta=0.85$

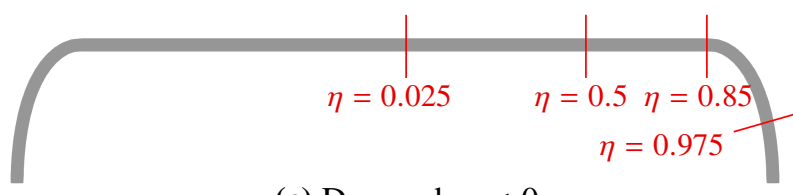

(c) Drooped, $q<0$

Fig. 6 Front views for (a) planar rectangular wing, (b) optimized design for $q>0$, and (c) optimized design for $q<0$. Specific sections are labeled in each case, which corresponds to the loading shown in Fig. 7 .

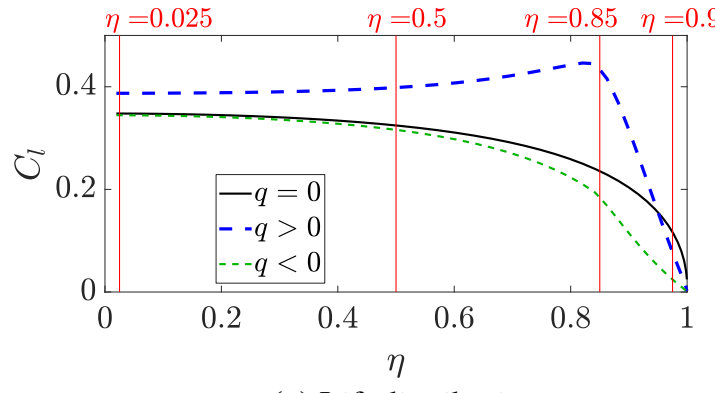

(a) Lift distribution

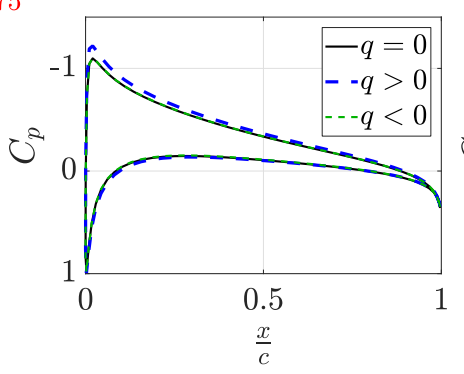

(c) $C_{p}$ at $\eta=0.025$

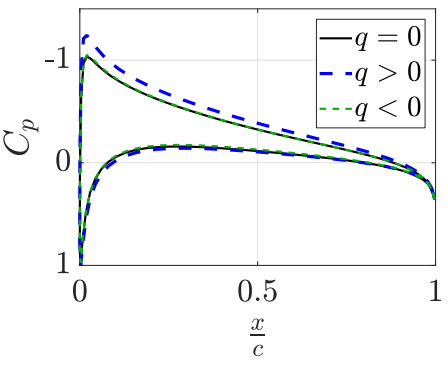

(d) $C_{p}$ at $\eta=0.5$

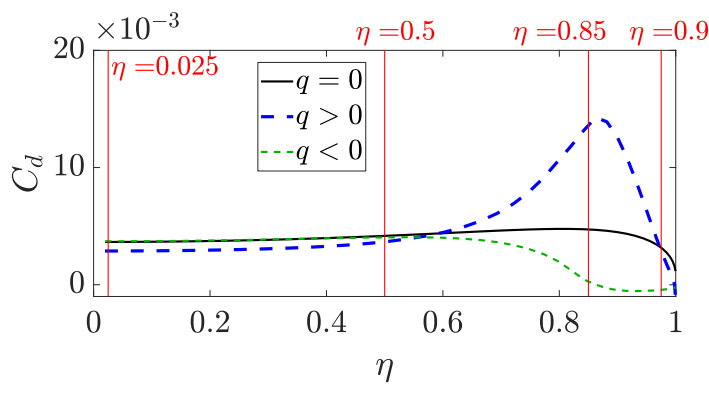

(b) Drag distribution

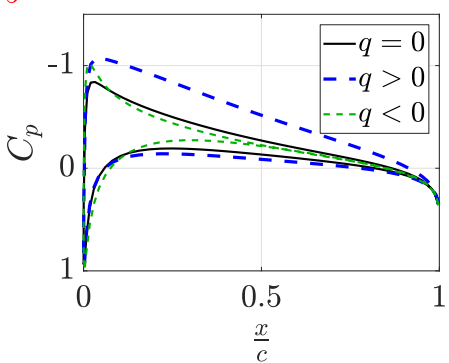

(e) $C_{p}$ at $\eta=0.85$

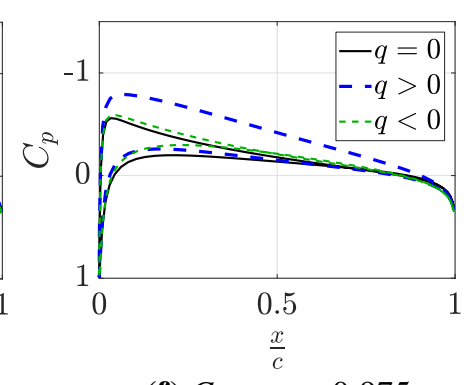

(f) $C_{p}$ at $\eta=0.975$

Fig. 7 Loading of wings shown in Fig. 6. (a) and (b) show spanwise lift and drag distributions respectively, where cross sectional $C_{p}$ distributions at the labeled $\eta$ locations is shown in (c)-(f).

\begin{tabular}{lc}
\hline \hline Wing & $e$ \\
\hline Planar, $q=0$ & 0.980 \\
Raised, $q>0$ & 1.138 \\
Drooped, $q<0$ & 1.219 \\
\hline \hline
\end{tabular}

Table 2 Efficiency factors for the rectangular planform wings shown in Fig. 6 
Both raised and drooped wings converge to the maximum wingtip bound on $q$. They also have a similar $q$-distribution where one is roughly the negative of the other. Both wings remain planar until $\eta \simeq 0.85$. However, there is a large difference in the efficiency factors, where compared to the rectangular planar wing, the drooped wing achieves a $24 \%$ increase, and the raised wing only a $16 \%$ increase.

The spanwise lift and drag distributions in Fig. $7 \mathrm{a}$-b provide a greater insight into these differences in efficiency between the three wing designs. When the wings are raised there is an induced lift along the span of the wing, but also an increase in drag towards the tip. As only induced drag is considered in the this study, the increase in drag is a direct result of the larger lift, both of which impact efficiency but with contrary effects. The drooped wing experiences the opposite to the raised, where the lift is reduced and there is a large reduction in drag towards the tip. A similar trend to the lift distributions can be seen in the accompanying $C_{P}$ distributions in Fig. 7k-f where the pressure difference has increased throughout the span for raised wings, and reduced towards the tip for drooped wings. The gains in efficiency from raised and drooped wings are due to achieving an increased lift and reduced drag respectively, but based on Fig. 7 alone it is not clear why this behavior is seen.

Using Cone's method of optimal spanwise loading [2] there should be no difference in efficiency between optimal raised and drooped wings for this optimization problem. The gain in performance of drooped wings is usually described as a result shifting the wingtip vortex core further outboard away from the wing [15]. However, this phenomena cannot be predicted by the current wake model, nor can it be predicted by the potential flow based models used in many of the studies presented in the literature that experienced an improved performance for drooped wings, e.g. [16, 18, 19, 29]. Liersch et al [19] offer an alternative explanation where the difference is due to effects of induced velocities parallel to the freestream, which can be demonstrated with the current model.

From the $C_{p}$ distributions shown Fig. 7 -f it is clear that the velocity across the upper surface has increased for raised wings, and at some sections, reduced for drooped wing. There is also a clear pressure difference between upper and lower surfaces in each case along the entire span of wing. Therefore, we know there is a circulation in the sections towards the wingtips which will induce a velocity. By raising or drooping the wing these induced velocities will behave differently. To demonstrate this, we define a scalar $\bar{V}$ as the velocity normalized by the freestream and projected in the freestream direction i.e.

$$
\bar{V}=\frac{\boldsymbol{V}}{\left\|\boldsymbol{V}_{\infty}\right\|} \cdot \frac{\boldsymbol{V}_{\infty}}{\left\|\boldsymbol{V}_{\infty}\right\|}=\frac{1}{\left\|\boldsymbol{V}_{\infty}\right\|^{2}} \boldsymbol{V} \cdot \boldsymbol{V}_{\infty}
$$

Fig. 8 shows $\bar{V}$ within a cut plane at the quarter point, $\frac{x}{c}=0.25$, for planar, raised and drooped wings. The raised wing has significantly increased the velocity in the freestream direction along the entire upper surface. This corresponds with an increase in lift seen in Fig. $7 \mathrm{~g}$ which peaks when the wing begins to cant upwards, also corresponding to the largest velocity in Fig. 8 For the drooped wing, the inboard velocities are very similar to the planar wing. Towards the tip the induced velocities are reduced (compared to the planar wing) on the upper surface and are larger on the lower 
surface. As the difference between upper and lower surfaces reduces so too does the pressure difference, and both lift and drag is significantly reduced.
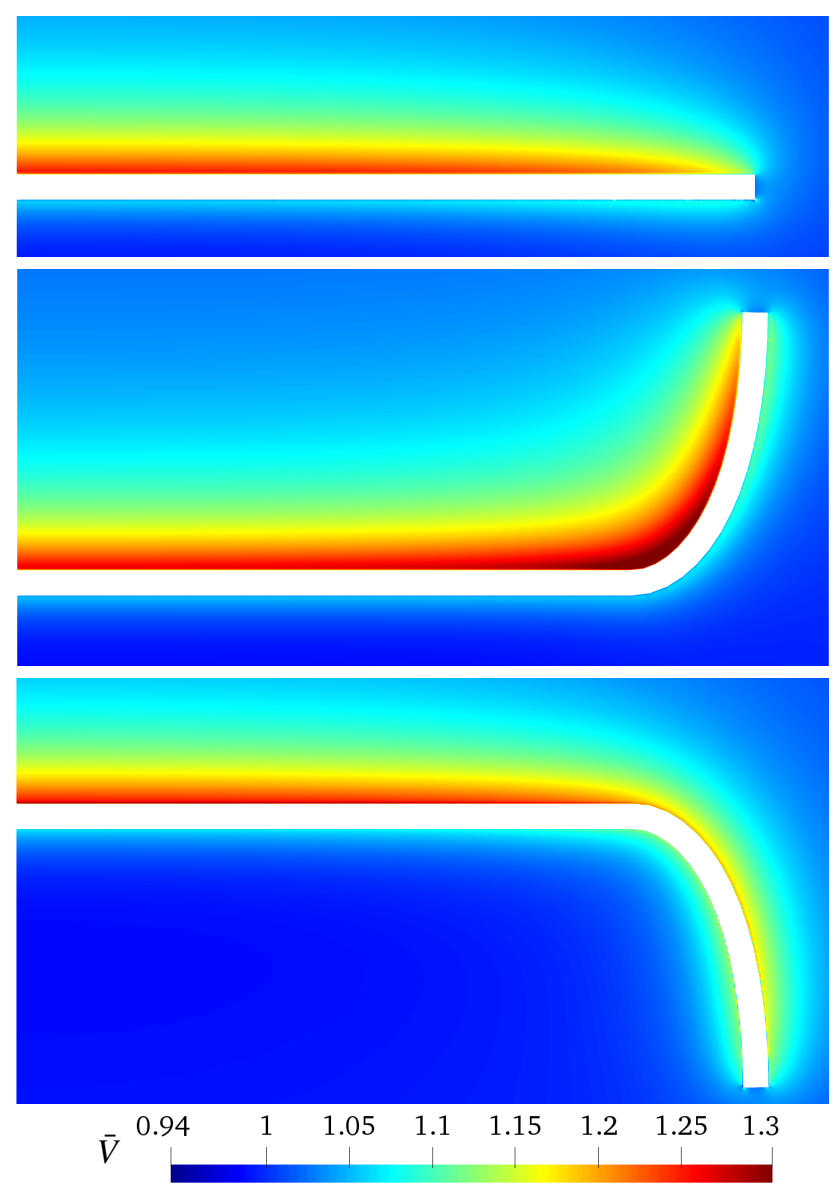

Fig. 8 Normalized velocity projected in the freestream direction, $\bar{V}=\frac{1}{\left\|V_{\infty}\right\|^{2}} V \cdot V_{\infty}$, for planar (top), raised (middle), and drooped (bottom) rectangular wings. Cut plane defined at quarter chord point.

The induced drag is directly related to the induced downwash in the wake. Off-stream velocity vectors in the Trefftz plane are shown in Fig. 9 for the planar, raised, and drooped wings. As expected the planar wing has a distinct circulation around the tip of the wake. When the wing is raised, the flow above the wake in region $0.5<\eta<1$ has a larger vertical component than is seen for the planar wing. This corresponds to a larger downwash which gives the increase in drag in Fig. 7p. For both the planar and raised wings, there is a downwash everywhere in the flow for $\eta<1$, i.e. the vertical component of velocity is negative. When the wing is drooped, there is a positive vertical component to the velocity above the wake in the region $0.85<\eta<1$. In the same region, the velocity below the wake is negative, meaning the potential jump (or downwash) over the wake should be close to 0 , thus producing very little drag. This is also clearly seen in this region in Fig. 7p. Note that the vectors in Fig. 9 indicate the velocity direction only, and the vector magnitudes do not represent the magnitude of velocity.

From Table 2 one would assume that drooped wings are superior to raised wings as they can create a larger gain in 


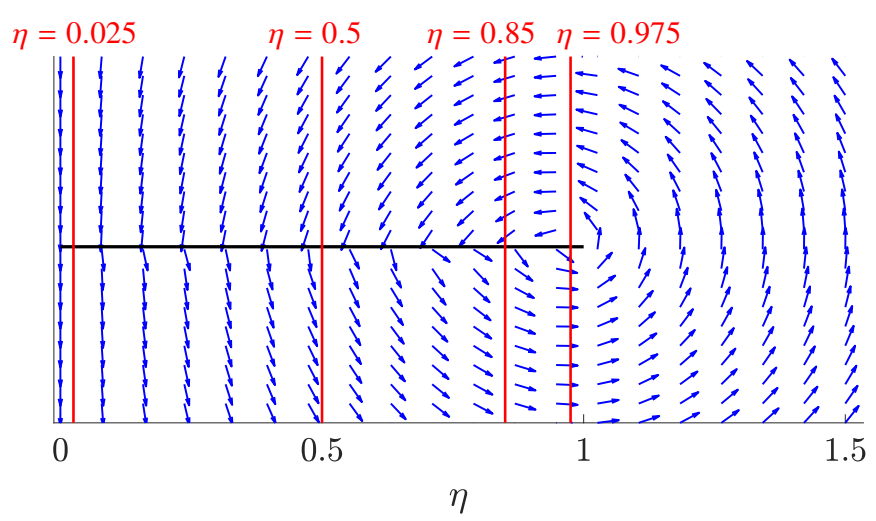

(a) Planar, $q=0$

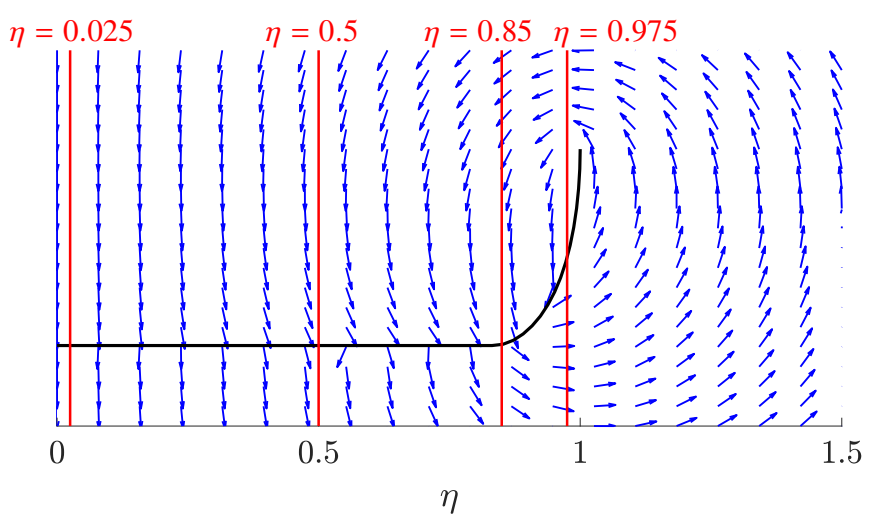

(b) Raised, $q>0$

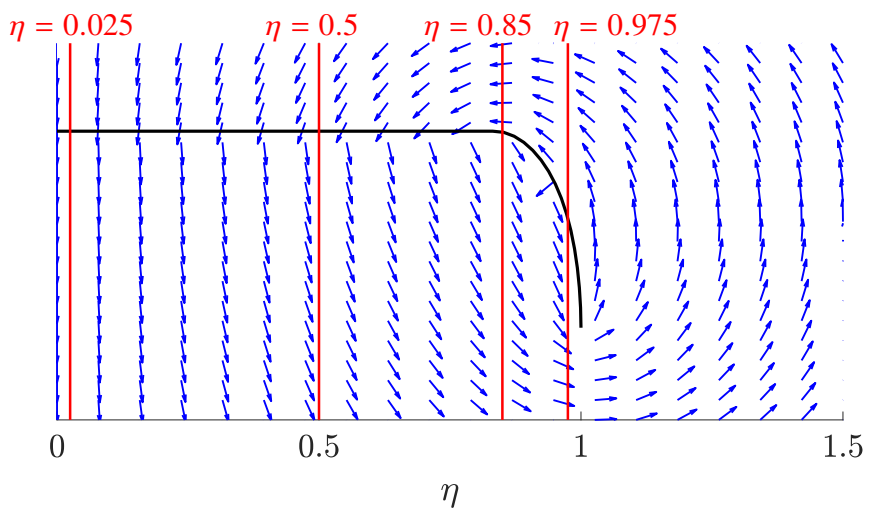

(c) Drooped, $q<0$

Fig. 9 Velocity vectors in the Trefftz plane for (a) planar, (b) raised, and (c) drooped wings. Vectors are used to indicate direction only, where vector magnitudes are not representative of velocity magnitudes.

efficiency. However, the optimization study in 17 has a restricted design space as only $q$ was allowed to change and the chord distribution was fixed. In most practical designs the wing will taper towards the tip, which in the case for raised wings would alleviate some the increased drag experienced towards the tip in Fig. 7p. The optimization problem in (13) is solved again for both raised and drooped wings with $\mathbf{x}=\{\delta \boldsymbol{c}, \delta \boldsymbol{a}, \delta \boldsymbol{q}\}$ and different values of $\bar{q}_{t}$, where $q_{0}$ is the original value of $\bar{q}_{t}$ from previous studies. Optimized designs are shown in Fig. 10 with lift and drag distributions shown in Fig. 11 and efficiency factors are given in Table 3 


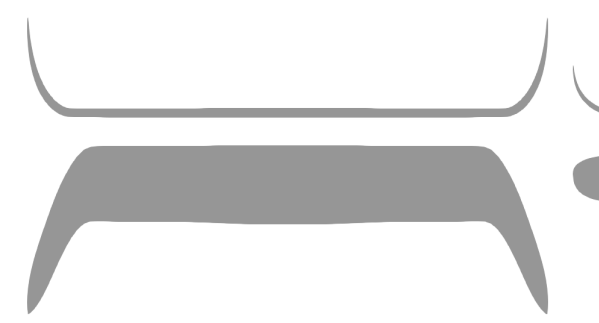
(a) $q>0, \bar{q}_{t}=q_{0}$
(b) $q>0, \bar{q}_{t}=\frac{1}{2} q_{0}$
(c) $q>0, \bar{q}_{t}=\frac{1}{4} q_{0}$

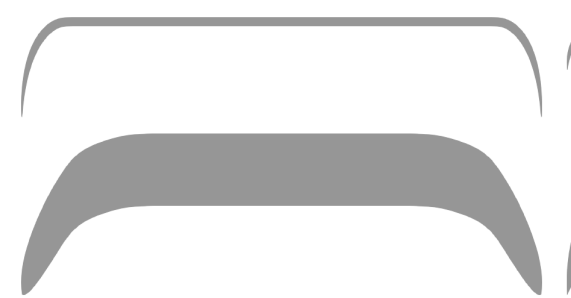
(d) $q<0, \bar{q}_{t}=q_{0}$
(e) $q<0, \bar{q}_{t}=\frac{1}{2} q_{0}$
(f) $q<0, \bar{q}_{t}=\frac{1}{4} q_{0}$

Fig. 10 Elevation and plan views of optimized designs for raised $(q>0)$ and drooped $(q<0)$ wings with different bounds on $q_{t}$.

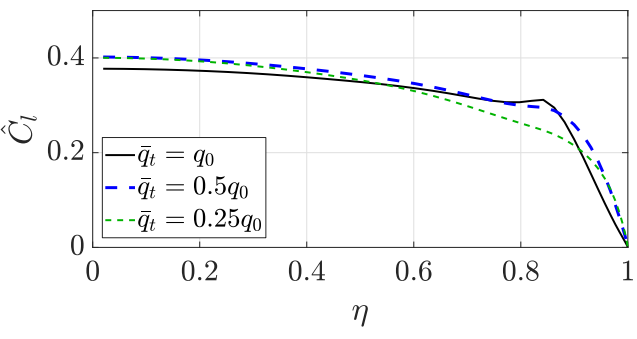

(a) $q>0$

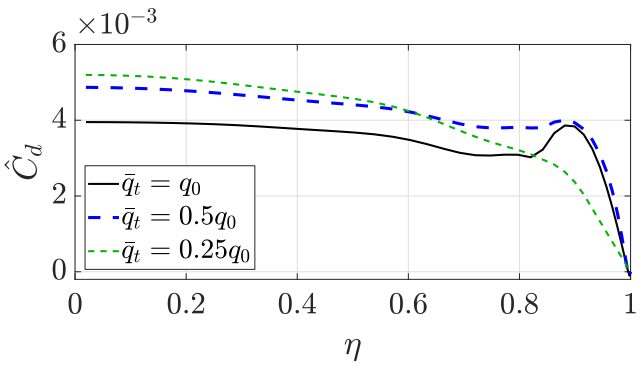

(c) $q>0$

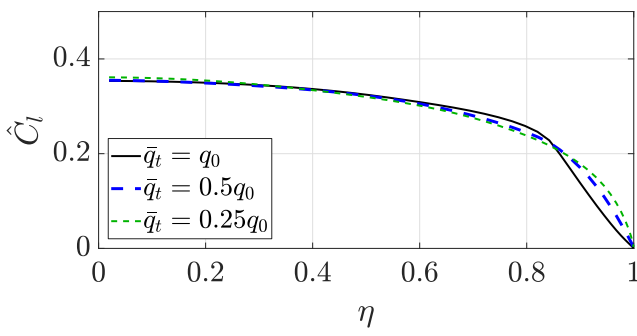

(b) $q<0$

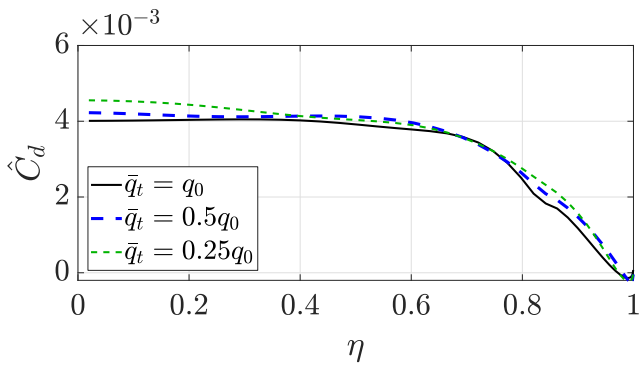

(d) $q<0$

Fig. 11 Lift and drag distributions for designs shown in Fig. 10, where lift and drag coefficients represented with a circumflex are calculated using the mean aerodynamic chord.

\begin{tabular}{lll}
\hline \hline $\bar{q}_{t}$ & $q>0$ & $q<0$ \\
\hline$q_{0}$ & 1.290 & 1.291 \\
$\frac{1}{2} q_{0}$ & 1.152 & 1.157 \\
$\frac{1}{4} q_{0}$ & 1.074 & 1.078 \\
\hline \hline
\end{tabular}

Table 3 Efficiency factors for the designs shown in Fig. 10 . 
As shown in Table 3, drooped and raised wings achieve efficiency factors with negligible differences for a given bound on $q_{t}$. This study has increased the design space such that the maximum efficiency can be achieved irrespective of whether the wings are raised or drooped. The raised wings in this case are able to match the performance of the drooped wings by reducing the peak in drag towards the tip by tapering the wing, compare Figs 11 p and $7 \mathrm{p}$. Drooped designs have created sweep which has the effect of increasing lift towards the tip [30] (Fig. 11]), but this also eliminates the low drag region seen in Fig. 7p. The optimizer has then created a trade-off between increasing lift and decreasing drag in order to maximize $e$.

Each drooped wing in Fig. 10 has a similar planform, but with small differences in $c$ and $a$ distributions where lower bounds on $q_{t}$ have a shorter chord length at the tip, and larger bounds on $q_{t}$ have a more gradual change in $a$. When the wings are raised with a large bound on $q_{t}$ the design converges to a similar planform to that of the drooped wings but with a larger sweep towards the tip. As the constraint on $q_{t}$ is restricted one would expect the converged designs to tend towards the equivalent design for a planar wing, shown in Fig. 4e. The drooped wing in Fig. 10f does have a similar planform, but the raised wing designs tend towards the elliptic wing. This suggests that large sweep can have negative effects for raised wingtips. To demonstrate this, analysis was conducted on the designs in Fig. 10 where they are solved upside-down, and efficiency factors are presented in Table 4

\begin{tabular}{lcc}
\hline \hline $\bar{q}_{t}$ & Opt. $q>0$ & Opt. $q<0$ \\
\hline$q_{0}$ & 1.267 & 1.267 \\
$\frac{1}{2} q_{0}$ & 1.118 & 1.109 \\
$\frac{1}{4} q_{0}$ & 1.051 & 1.039 \\
\hline \hline
\end{tabular}

Table 4 Efficiency factors for the designs shown in Fig. 10, when upside-down.

All wings see a reduction in $e$ when solved upside-down. As stated previously, $e$ should be constant irrespective of angle of attack, but they have changed when the sign on $\alpha$ has changed. This is due to differences in induced velocities when the wing is raised or drooped as discussed previously. For large values of $q_{t}$ there is no difference in $e$ between designs optimized for raised or drooped wings. For low $q_{t}$ the designs with large-raked wingtips perform worse when the wing is raised relative to the freestream (i.e. design optimized for drooped wings). To further demonstrate the effect of sweep on optimized designs, the problem in 17 is solved with no sweep, i.e. $\mathbf{x}=\{\boldsymbol{c}, \boldsymbol{q}\}$, where $\bar{q}_{t}=\frac{1}{4} q_{0}$, and result are shown in Fig. 12. Both raised and drooped wings satisfy the maximum bound of $q_{t}$, and produce efficiency factors of 1.074 and 1.055 respectively. Compared to the designs where sweep was included (Fig. 10) the raised wing converges to the same design, with the same efficiency, but the efficiency of the drooped wing has decreased significantly.

In the introduction it was discussed how the literature has contradicting results on whether raised or drooped wings can outperform one another. Tables 24 give similar contradictions which arise based on how the design space is restricted. Allowing a large design space achieves designs with negligible differences in $e$ between raised or drooped 


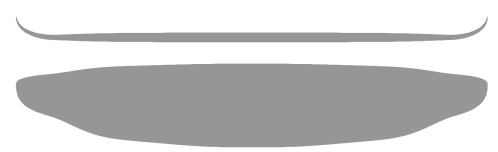

(a)

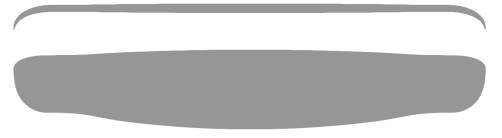

(b)

Fig. 12 Elevation and plan views of unswept optimized designs for (a) raised and (b) drooped wings.

wings with the same geometry restrictions. Results also presented here suggest not only that including sweep is crucial for improving performance in drooped but it can also reduce performance in raised wings. However, up to now the design space has also been somewhat restricted as twist has not yet been considered as a design variable, which grants more freedom to vary the span loading.

The literature also contains an important point of discussion on how the aspect ratio is defined when comparing the efficiency of planar and non-planar wings. In the previous examples $R$ is defined using the projected wingspan and planform area, as this is the standard definition. However, in using this definition it is not surprising that a non-planar wing can produce a large improvement in efficiency since the lifting surface has been increased without cost to the optimization problem. An alternative approach is to define these parameters using the unfolded wing geometry based on the curved length of the wing. When comparing planar and non-planar wings this difference in the geometry definition produces very different results and has been discussed in detail in [19, 31]. When solving the optimization problem with the aspect ratio defined from the unfolded wing geometry the designs converge to the same planar wings in Fig. $4 \mathrm{~d}$ or 4 e depending on whether $a$ is included as a design variable. In general, we note that if the curved geometry is constrained, the design will always converge to a planar wing with the maximum allowable wingspan. However, in aircraft design there is usually a constraint on the wingspan due to structural or practical reasons. With this constraint satisfied, the wing's performance can be improved by creating non-planar geometry.

\section{Optimization with Inviscid \& Viscous Drag}

Creating non-planar geometry may increase $e$, but the surface area will also increase which will increase the viscous drag. Referring back to the designs presented in Section IV.A their performance can also be compared with respect to drag coefficients and lift-to-drag ratio, as shown in Fig. 13, where the optimized design refers to the case with $\mathbf{x}=\{\delta \boldsymbol{c}, \delta \boldsymbol{a}, \delta \boldsymbol{q}\}$, shown in Fig. 4f, and all problems are solved with a freestream velocity of Mach 0.4. Fig. 13a plots both inviscid and viscous drag, where the design is optimized with respect to only inviscid drag. The optimized design produces the least inviscid drag for all values of $C_{L}$, but produces more viscous drag at low $C_{L}$ values where the viscous drag is dominant. This is evident also in Fig. $13 \mathrm{p}$ where the lift-to-drag ratio is lower for low $C_{L}$ values, where the optimized wing produced a $C_{L}$ value of $\sim 0.3$. However, each wing produces a similar maximum ${ }^{L} / D$ with the optimized design at 32.84 compared to 32.48 and 32.55 with HECS wings $p=2.5$ and $p=6$ respectively.

Consider the optimization problem given in 19$]$, to maximize ${ }^{L} / D$ at a specified $C_{L}$, fixed aspect ratio, the same 


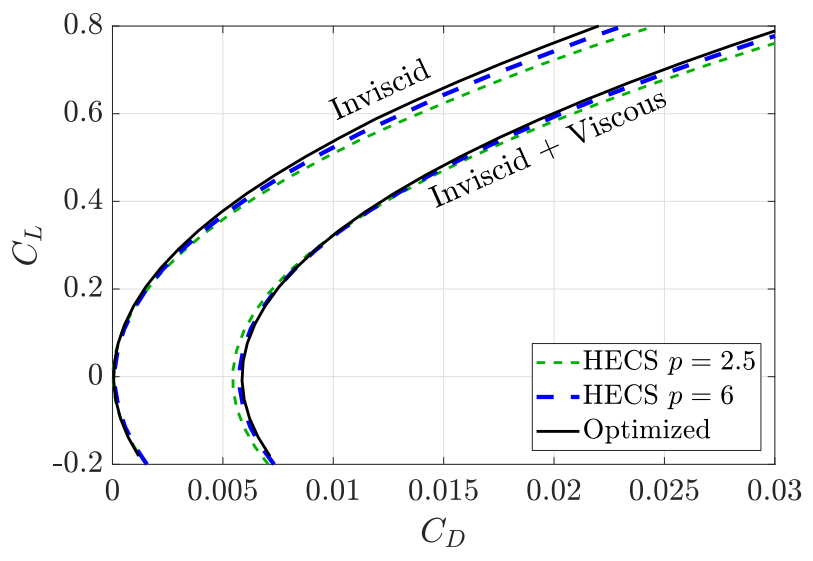

(a)

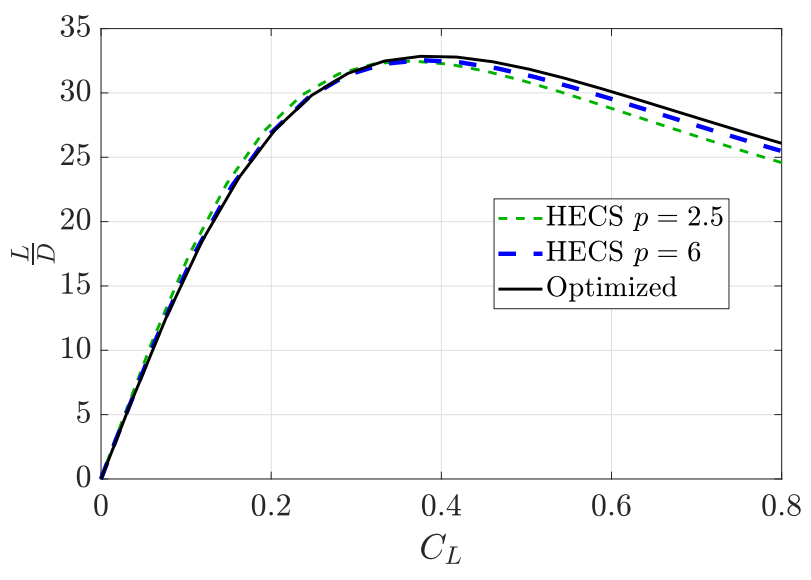

(b)

Fig. 13 Comparison between different wings: (a) drag coefficient for a range of lift coefficients for inviscid drag alone and inviscid plus viscous drag, (b) lift-to-drag ratio for viscous solutions.

geometry constraint on $a_{t}$, and $\bar{q}_{t}=\frac{1}{2} q_{0}$. The problem is solved for both raised and drooped wings, with multiple specified values of $C_{L}$. In each case, the problem is also solved where only induced drag is consider and where induced and viscous drag is considered. Each problem includes all design variables, i.e. $\mathbf{x}=\{\delta \boldsymbol{c}, \delta \boldsymbol{a}, \delta \boldsymbol{q}, \boldsymbol{\theta}\}$, where angle of attack is fixed at 0 degs. Note also that by specifying the $C_{L}$ and $A R$ the lift is also specified and maximizing ${ }^{L} / D$ is equivalent to minimizing $D$. Results are shown in Figs 14 and 15 for drooped and raised wings respectively with $L / D$ values shown in Table 5

$$
\begin{array}{ll}
\min _{\mathbf{x} \in \mathbb{R}^{N_{\mathrm{x}}}} & :-\frac{L}{D}(\mathbf{x}, \boldsymbol{\mu}) \\
\text { subject to } & : A R(\mathbf{x})=\AA_{0} \\
& C_{L}(\mathbf{x}, \boldsymbol{\mu})=C_{L_{0}} \\
& \left|a_{t}(\mathbf{x})\right| \leq \bar{a}_{t} \\
& \left|q_{t}(\mathbf{x})\right| \leq \bar{q}_{t} \\
& \underline{\mathbf{x}} \leq \mathrm{x}_{j} \leq \overline{\mathrm{x}} \quad \text { for } j=1, \ldots, N_{\mathrm{x}}
\end{array}
$$

For purely inviscid analysis, each of the raised and drooped wings converged to similar raked wingtip designs where constraints on $a_{t}$ and $q_{t}$ are active. In previous problems, the raised wing designs with the same bound on $q_{t}$ converged to wings which were almost elliptic (refer to $10 \mathrm{p}$ ). This difference is likely due to the inclusion of twist as a design variable which offers more design freedom to tailor the spanwise load distribution.

Considering cases with only inviscid analysis, there are negligible differences in converged designs with different $C_{L}$-values - for both raised and drooped wings. When viscous drag is considered and $C_{L_{0}}=0.2$, the constraint on $q$ is not active in either case. The low $C_{L}$ value means that the induced drag is low as it will tend to 0 for $C_{L}=0$. Because 


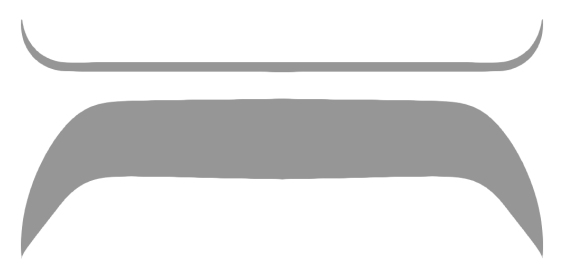

(a) $D=D_{i}, C_{L_{0}}=0.2$

(b) $D=D_{i}, C_{L_{0}}=0.4$

(c) $D=D_{i}, C_{L_{0}}=0.6$
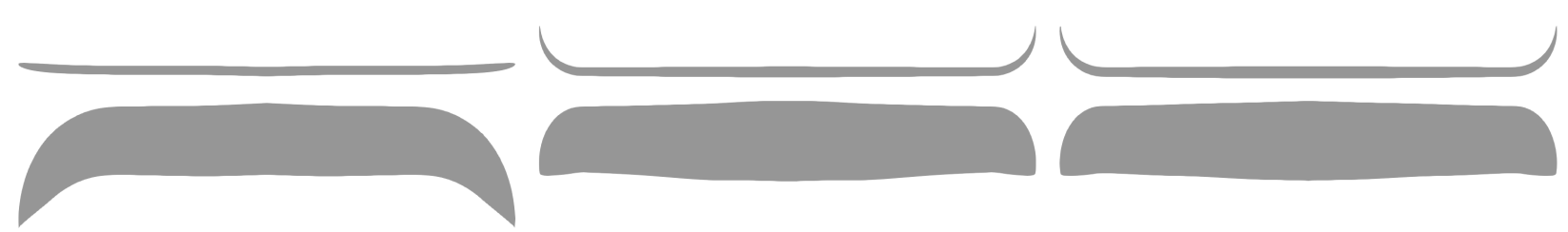

(d) $D=D_{i}+D_{v}, C_{L_{0}}=0.2$

(e) $D=D_{i}+D_{v}, C_{L_{0}}=0.4$

(f) $D=D_{i}+D_{v}, C_{L_{0}}=0.6$

Fig. 14 Elevation and planform views for converged raised wing designs with (a)-(c) inviscid analysis and (d)-(f) viscous analysis, for different specified values of $C_{L}$.

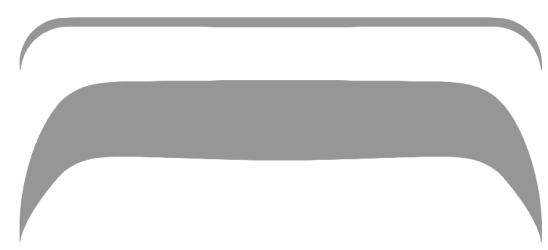

(a) $D=D_{i}, C_{L_{0}}=0.2$
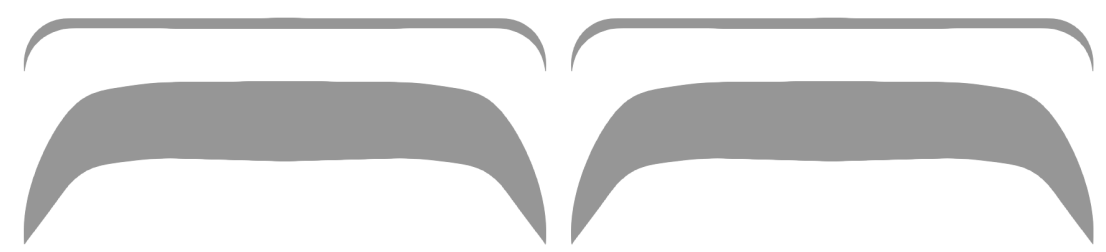

(b) $D=D_{i}, C_{L_{0}}=0.4$

(c) $D=D_{i}, C_{L_{0}}=0.6$
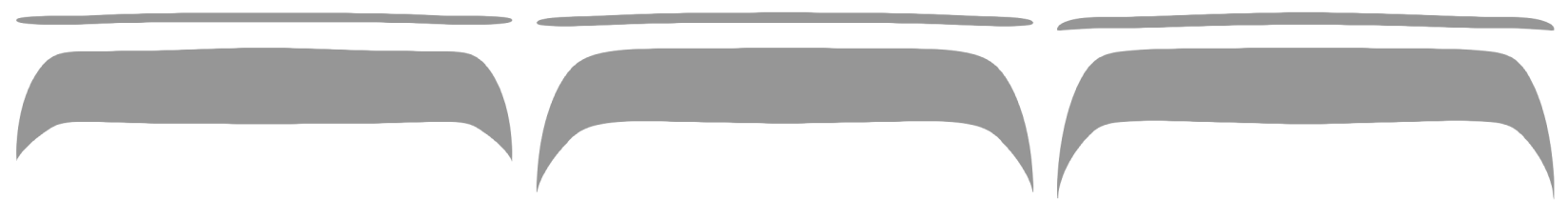

(d) $D=D_{i}+D_{v}, C_{L_{0}}=0.2$

(e) $D=D_{i}+D_{v}, C_{L_{0}}=0.4$

(f) $D=D_{i}+D_{v}, C_{L_{0}}=0.6$

Fig. 15 Elevation and planform views for converged drooped wing designs with (a)-(c) inviscid analysis and (d)-(f) viscous analysis, for different specified values of $C_{L}$.

\begin{tabular}{cccccc}
\hline \hline$D$ & $C_{L_{0}}$ & $\begin{array}{c}L / D \\
q>0\end{array}$ & $\begin{array}{c}L / D \\
q<0\end{array}$ & $\begin{array}{c}\frac{D_{i}}{D_{i}+D_{v}} \\
q>0\end{array}$ & $\begin{array}{c}\frac{D_{i}}{D_{i}+D_{v}} \\
q<0\end{array}$ \\
\hline$D_{i}$ & 0.2 & 124.1 & 123.6 & 1.0 & 1.0 \\
$D_{i}$ & 0.4 & 62.9 & 62.1 & 1.0 & 1.0 \\
$D_{i}$ & 0.6 & 42.5 & 42.1 & 1.0 & 1.0 \\
$D_{i}+D_{v}$ & 0.2 & 29.7 & 29.6 & 0.256 & 0.257 \\
$D_{i}+D_{v}$ & 0.4 & 35.1 & 33.7 & 0.521 & 0.555 \\
$D_{i}+D_{v}$ & 0.6 & 31.5 & 29.6 & 0.685 & 0.648 \\
\hline \hline
\end{tabular}

Table 5 Lift-to-drag ratios for wings optimized at different $C_{L}$ values with and without viscous effects, and ratio of induced drag to total drag. 
the viscous drag does not tend to zero, it is dominant in these designs where $C_{L}$ is low, refer to Table 5 columns 5 and 6. Because the viscous drag is dominant, it is not beneficial to increase $q$ as to do so will increase $D_{v}$.

When $C_{L_{0}}$ is increased the induced drag increases much faster than the viscous drag. This makes it beneficial for raised wing designs to create large winglets as to do so will increase the lift. Designs for $q<0$ do not create drooped wings when viscosity is considered for any value of $C_{L_{0}}$. When a larger $C_{L}$ is specified, there is a large change in $a$ towards the wingtip where designs converge to wings similar to the optimized swept planar wing in Fig. 4e. Increasing $a$ in this manner improves the inviscid efficiency, as demonstrated previously. The fact that drooped wings did not form suggests that the reduction in induced drag as a results of drooping the wing is less than the increase in viscous drag that will also be created.

For each inviscid case, raised and drooped wings produced similar lift-to-drag ratios. This is in keeping with results presented in the previous section where the same $e$-factors could be achieved regardless of whether wings are raised or drooped. When viscous effects are considered, similar designs are produced for $C_{L_{0}}=0.2$, with similar lift-to-drag ratios. When $C_{L_{0}}$ is increased, the raised wings outperform designs with $q<0$.

Fig. 16 shows the lift and drag relationships for designs optimized with viscous effects for $C_{L_{0}}=\{0.2,0.6\}$. Optimizing with larger $C_{L}$ values has produced designs with a larger maximum lift-to-drag ratio. The lift-to-drag ratio is highest for raised wing designs with $C_{L_{0}}=0.6$, at 34.9, compared to 33.7 with $q<0$ and 33.1 for both designs with $C_{L_{0}}=0.2$.

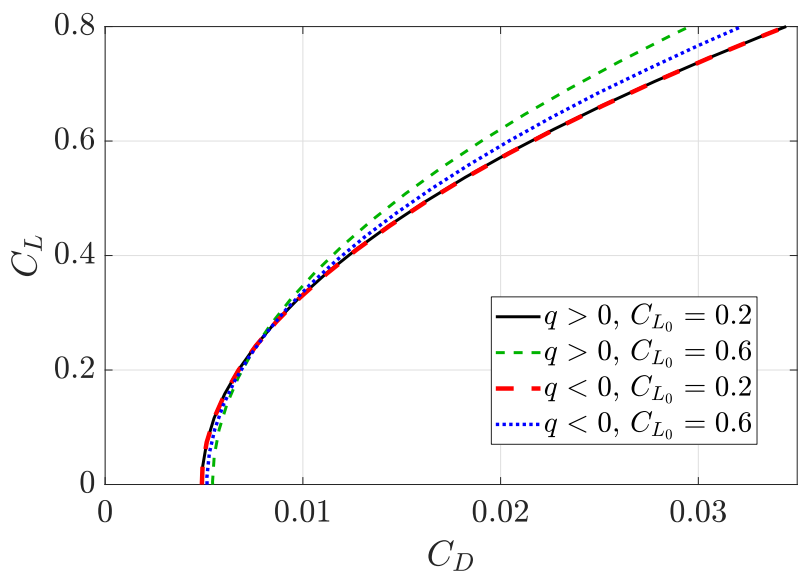

(a)

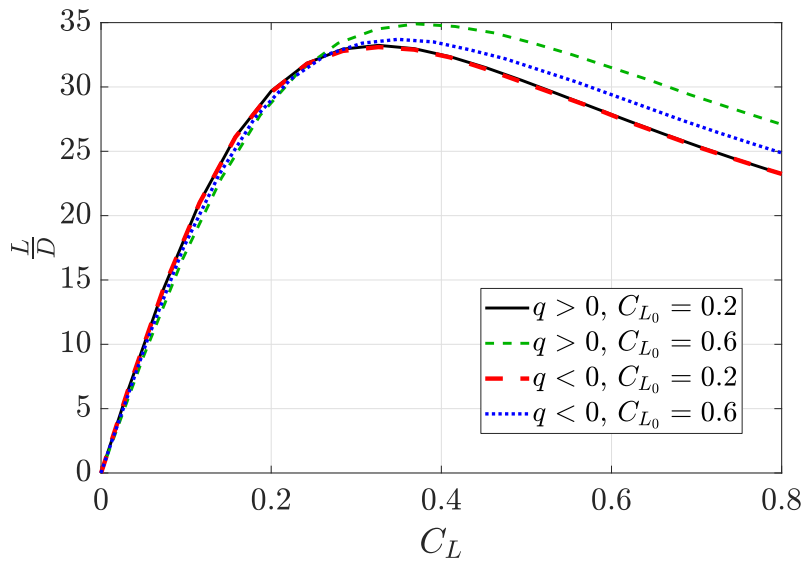

(b)

Fig. 16 Lift and drag properties for converged designs with viscous calculations in Figs 14 and 15 : (a) drag coefficient and (b) lift-to-drag ratio, for a range of lift coefficients.

It is worth highlighting that even though results suggest that drooped wings are not beneficial when viscous analysis is considered, we have not accounted for structural deformations. There is however evidence that drooped wings can be beneficial in viscous flow when aeroelastic analysis is considered, e.g. [17]. It is also important to note that when minimizing the sum of induced and viscous drag, the optimizer may choose to minimize one over the other. This can 
cause the optimizer to converge to local minima with irregular geometry and equal or worse performance. In order to avoid this bias, it is important to start with a reasonable initial design. As stated previously, each optimization problem presented in this work starts with an initial design of a rectangular wing with no twist and all constraints satisfied. However, similar results have also been achieved with other common planar wing designs such as tapered or elliptic wings. Multiple solutions and local minima for these types of optimization problems with a large design space have also been discussed in the past [32].

Lift-to-drag gradients of the initial design are shown in Fig. 17 for inviscid and viscous analysis with $C_{L}=0.6$. The wing is most sensitive to changes in twist and chord, especially for inviscid analysis. When viscous drag is included there is less variance in the gradients both for different design variables and for the spanwise location. The gradients of $q$ which controls the non-planar geometry also approaches zero, apart from at the tip where the gradient's magnitude increases drastically in each case. Convergence history of the cases with $C_{L_{0}}=0.6$ are shown in Fig. 18 . The behavior shown in this plot is also representative of other cases, where typically the problem takes longer to converge for drooped wing designs and when viscous analysis is considered.

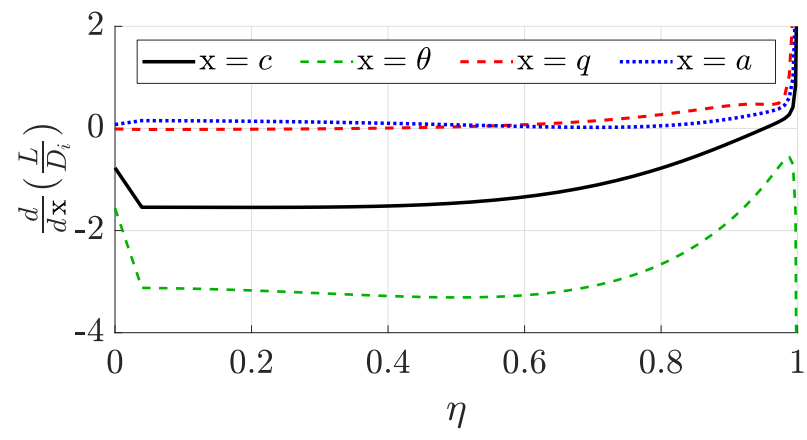

(a)

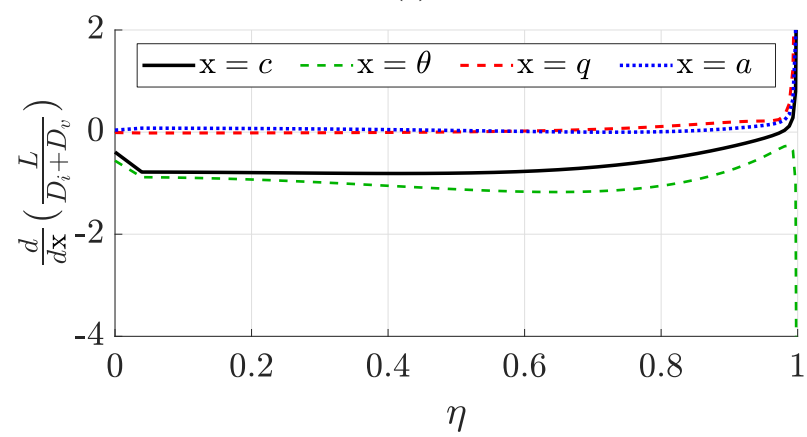

(b)

Fig. 17 Lift to drag gradients of the initial design considering design variables $\mathbf{x}=\{c, \theta, q, a\}$, for (a) inviscid analysis and (b) viscous analysis. Note that in each case the tip value $(\eta=1)$ is not shown in the current range. 


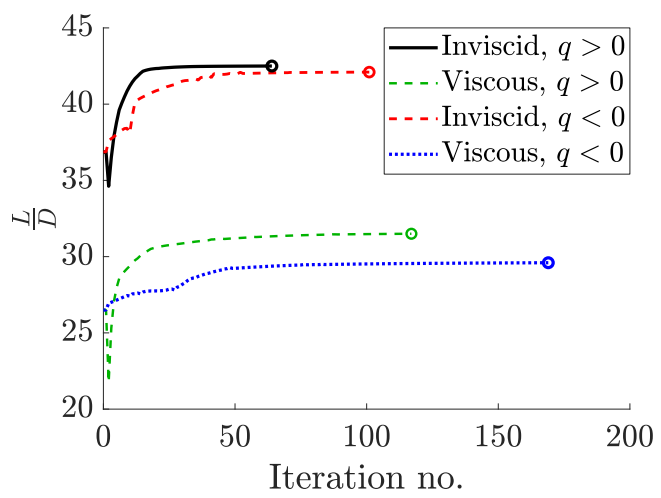

Fig. 18 Convergence history with inviscid and viscous analysis for raised and drooped designs with $C_{L_{0}}=0.6$ (refer to Figs 14 and 15 .

\section{Conclusions}

An optimization procedure is introduced for non-planar wings where design variables are defined locally in order to enlarge the design space. By increasing the design space, the optimizer is able to create large changes to the geometry in order to achieve optimized designs. This is the main advantage of the method which has been able to create designs that predict improved performance over reference wings from literature. The aerodynamic improvements of drooped wings is commonly explained by the effect of shifting the wingtip vortex core further outboard. Results presented here have shown that the benefits of drooped wings can be captured with fixed wake models. It has been demonstrated that the predicted improvements in aerodynamic performance for both raised and drooped wings was due to the effects of induced velocities parallel to freestream, which behave differently depending on whether the wing is raised or drooped. Studies presented here also contribute to an ongoing discussion in literature of whether drooped wings are more efficient than raised - to which there is no general consensus in the literature. Here it has been shown that optimization studies can yield conflicting results depending on how the optimization problem is posed. Ultimately, with a large enough design space, raised and drooped wings should be able to converge to designs with equal performance when only inviscid analysis is considered. When viscous approximations were included, drooped wings have not shown to be beneficial.

\section{Appendix}

\section{A. Impact of mesh discretization}

This appendix studies the effect of mesh discretization on results. Fig. 19 shows mesh convergence studies in chordand spanwise directions for the drooped HECS wing shown in Fig. 4f with SD7032 airfoils, (further details on the wing geometry are given in [14]). Results are not sensitive to discretization error in the spanwise direction but are sensitive to the chordwise discretization. For optimization problems presented in this work, a chordwise discretization of 100 panels was chosen as the relative error is less than $1 \%$ (refer to Fig. 19a). A spanwise discretization of 40 panels was chosen, not reduce the error, but because the design variables are defined at the intersection of spanwise panels where increasing 
the spanwise discretization allows for more design variables which increases the design space and can lead to larger changes in geometry.

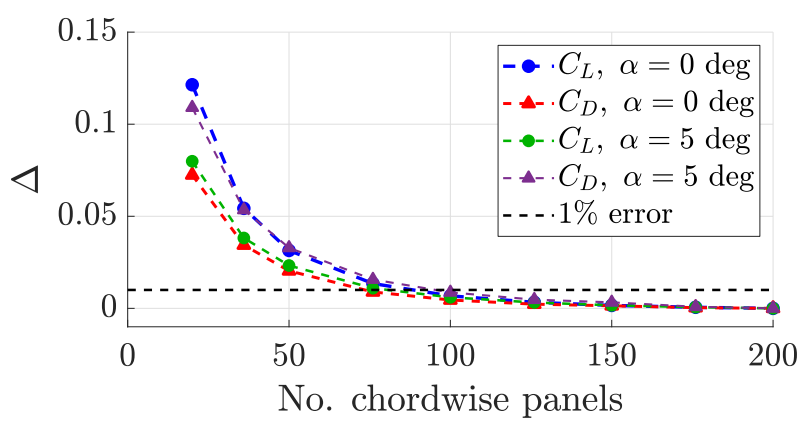

(a)

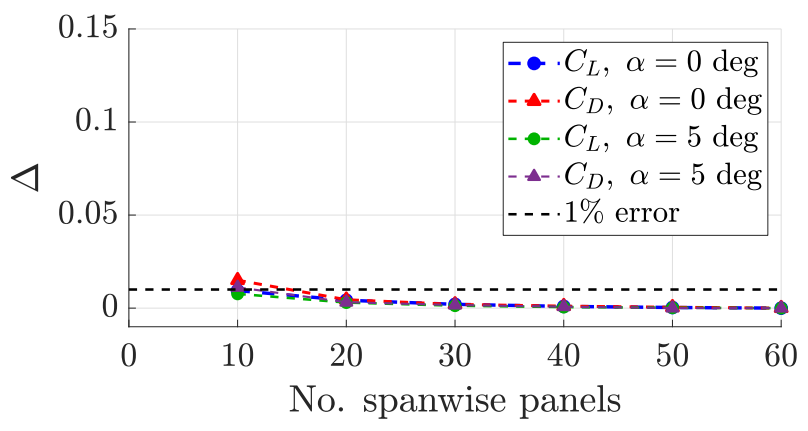

(b)

Fig. 19 Mesh convergence studies for a drooped HECS wing: (a) chordwise mesh convergence with 40 spanwise panels and (b) spanwise mesh convergence with 100 chordwise panels. Parameter $\Delta$ refers to the relative error.

\section{B. Comparison to Experimental Data}

To demonstrate the method presented in this work, numerical results for a drooped HECS wing are compared to the experimental data provided in [14]. Planform and elevation views of the wing are shown in Fig. 4k, where the wing is formed from SD7032 airfoils (specific details on the wing definition are provided in [14]). Fig. 203 the wing's lift polar where there are negligible differences between numerical and experimental data. Because the numerical model cannot predict stall, the results start to diverge for large angles of attack ( $>10 \mathrm{degs})$, however this is outside the range of operating conditions studied in this work. Comparing results in Fig. 20p the data is also in close agreement for high $C_{L}$ values where the induced drag is dominant but diverges at low $C_{L}$ values when the viscous drag is dominant. This is

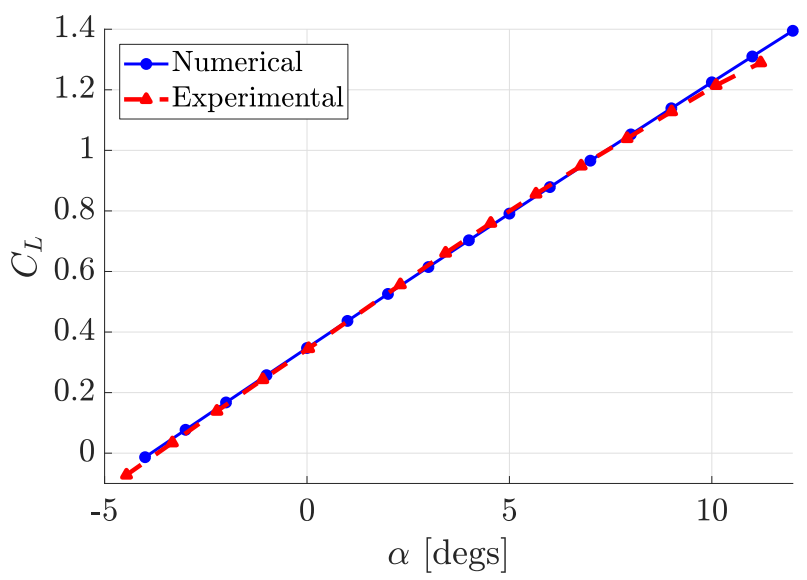

(a)

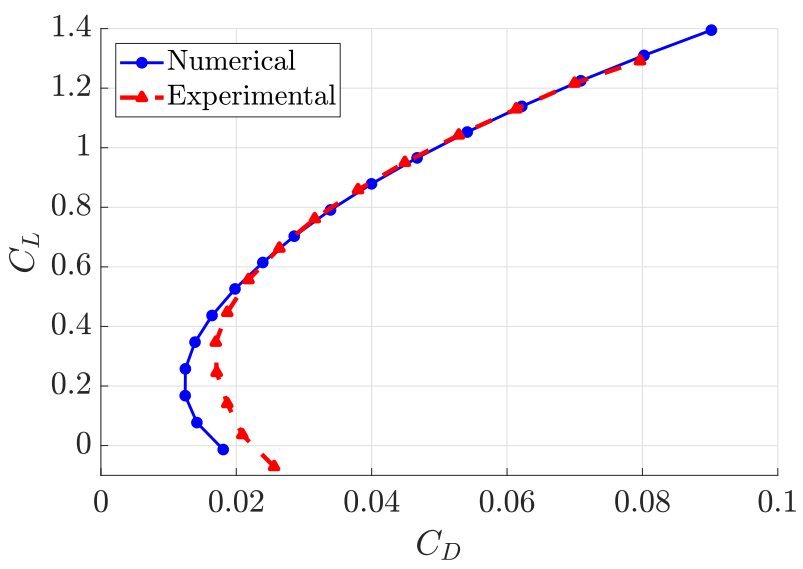

(b)

Fig. 20 Comparison between numerical and experimental results for a drooped HECS wing: (a) freestream angle of attack vs. lift coefficient and (b) drag coefficient for a range of lift coefficients. 
likely due to the fact that viscous drag is calculated based on 2D airfoils sections which neglects the spanwise flow. However, it is noted that the numerical model still predicts a similar trend to the experimental results in this low $C_{L}$ region.

\section{Acknowledgments}

The authors would like to acknowledge and thank the Villum Foundation for the financial support provided through the InnoTop Villum Investigator project.

\section{References}

[1] Munk, M. M., The Minimum Induced Drag of Aerofoils, NASA Technical Report TR-121, National Aeronautics and Space Administration, 1923.

[2] Cone, C. D. J., The Theory of Induced Lift and Minimum Induced Drag of Nonplanar Lifting Systems, NASA Technical Report TR-139, National Aeronautics and Space Administration, 1962.

[3] Demasi, L., "Induced drag minimization: A variational approach using the acceleration potential," Journal of Aircraft, Vol. 43, No. 3, 2006, pp. 669-680. doi:https://doi.org/10.2514/1.15982.

[4] Demasi, L., "Investigation on conditions of minimum induced drag of closed wing systems and C-wings," Journal of Aircraft, Vol. 44, No. 1, 2007, pp. 81-99. doi:https://doi.org/10.2514/1.21884.

[5] Demasi, L., Dipace, A., Monegato, G., and Cavallaro, R., "Invariant formulation for the minimum induced drag conditions of nonplanar wing systems," Aiaa Journal, Vol. 52, No. 10, 2014, pp. 2223-2240. doi:https://doi.org/10.2514/1.J052837.

[6] Hicken, J. E., and Zingg, D. W., "Induced-drag minimization of nonplanar geometries based on the Euler equations," Aiaa Journal, Vol. 48, No. 11, 2010, pp. 2564-2575. doi:https://doi.org/10.2514/1.J050379.

[7] Gagnon, H., and Zingg, D. W., "Euler-equation-based drag minimization of unconventional aircraft configurations," Journal of Aircraft, Vol. 53, No. 5, 2016, pp. 1361-1371. doi:https://doi.org/10.2514/1.C033591.

[8] Secco, N. R., and Martins, J. R., "RANS-based aerodynamic shape optimization of a strut-braced wing with overset meshes," AIAA/ASCE/AHS/ASC Structures, Structural Dynamics, and Materials Conference, 2018, , No. 210049, 2018 , p. 21 pp. doi:https://doi.org/10.2514/6.2018-0413.

[9] Maskew, B., Program VSAERO Theory Document : A Computer Program for Calculating Nonlinear Aerodynamic Characteristics of Arbitrary Configurations, NASA contractor report CR-4023, National Aeronautics and Space Administration, 1987.

[10] Erickson, L., Panel Methods - An Introduction, NASA Technical Paper 2995, National Aeronautics and Space Administration, 1990. 
[11] Conlan-Smith, C., Ramos-García, N., Sigmund, O., and Andreasen, C. S., "Aerodynamic Shape Optimization of Aircraft Wings Using Panel Methods,” AIAA Journal, Vol. 58, No. 9, 2020, pp. 3765-3776. doi:https://doi.org/10.2514/1.J058979.

[12] Conlan-Smith, C., and Andreasen, C. S., "Aeroelastic Optimization of Aircraft Wings Using a Coupled Three-Dimensional Panel-Beam Model,” AIAA Journal, Vol. 59, No. 4, 2021, pp. 1374-1386. doi:https://doi.org/10.2514/1.J059911.

[13] Van Dam, C., "Efficiency characteristics of crescent-shaped wings and caudal fins," Nature, Vol. 325, No. 6103, 1987, pp. 435-437. doi:https://doi.org/10.1038/325435a0.

[14] Lazos, B. S., "Biologically inspired fixed-wing configuration studies," Journal of Aircraft, Vol. 42, No. 5, 2005, pp. $1089-1098$. doi:https://doi.org/10.2514/1.10496.

[15] Lazos, B. S., and Visser, K. D., "Aerodynamic comparison of Hyper-Elliptic Cambered Span (HECS) wings with conventional configurations," Collection of Technical Papers - AIAA Applied Aerodynamics Conference, Vol. 3, 2006, pp. 1608-1625. doi:https://doi.org/110.2514/6.2006-3469.

[16] Nguyen, N., Trinh, K., Reynolds, K., Kless, J., Aftosmis, M., Urnes, J., and Ippolito, C., "Elastically shaped wing optimization and aircraft concept for improved cruise efficiency," 51st AIAA Aerospace Sciences Meeting Including the New Horizons Forum and Aerospace Exposition, 2013. doi:https://doi.org/10.2514/6.2013-141.

[17] Khosravi, S., and Zingg, D. W., “Aerostructural optimization of drooped wings," Journal of Aircraft, Vol. 55, No. 3, 2018 , pp. 1261-1268. doi:https://doi.org/10.2514/1.C034605.

[18] Ranjan, P., Ansell, P. J., and James, K. A., “Optimal hyperelliptic cambered span configurations for minimum drag," Journal of Aircraft, Vol. 56, No. 1, 2019, pp. 356-368. doi:https://doi.org/110.2514/1.C034992.

[19] Liersch, C. M., Streit, T., and Visser, K. D., "Numerical implications of spanwise camber on minimum induced drag configurations," 47th AIAA Aerospace Sciences Meeting Including the New Horizons Forum and Aerospace Exposition, 2009. doi:https://doi.org/10.2514/6.2009-898.

[20] Pomeroy, B. W., and Visser, K. D., "A computational study of induced drag behavior for spanwise cambered wings," 28th AIAA Applied Aerodynamics Conference, Vol. 1, 2010. doi:https://doi.org/10.2514/6.2010-4227.

[21] Ramos García, N., Sørensen, J., and Shen, W., “Three-Dimensional Viscous-Inviscid Coupling Method for Wind Turbine Computations," Wind Energy, Vol. 19, No. 1, 2016, p. 67-93. doi:https://doi.org/10.1002/we.1821.

[22] Katz, J., and Plotkin, A., Low-Speed Aerodynamics, Cambridge Aerospace Series, Cambridge University Press, 2001.

[23] Smith, S. C., A Computational and Experimental Study of Nonlinear Aspects of Induced Drag, Technical Paper 3598, National Aeronautics and Space Administration, 1996.

[24] Drela, M., Flight Vehicle Aerodynamics, The MIT Press, 2014. 
[25] Leishman, J. G., Bhagwat, M. J., and Bagai, A., "Free-Vortex Filament Methods for the Analysis of Helicopter Rotor Wakes," Journal of Aircraft, Vol. 39, No. 5, 2002, pp. 759-775. doi:https://doi.org/10.2514/2.3022.

[26] Drela, M., "XFOIL: An Analysis and Design System for Low Reynolds Number Airfoils," Low Reynolds Number Aerodynamics, edited by T. J. Mueller, Springer Berlin Heidelberg, Berlin, Heidelberg, 1989, pp. 1-12.

[27] Svanberg, K., “The Method of Moving Asymptotes - a New Method for Structural Optimization,” International Journal for Numerical Methods in Engineering, Vol. 24, No. 2, 1987, pp. 359-373. doi:https://doi.org/10.1002/nme.1620240207.

[28] Van Dam, C., Vijgen, P., and Holmes, B., "Experimental Investigation on the effect of crescent planform on lift and drag," Journal of Aircraft, Vol. 28, No. 11, 1991, pp. 713-720. doi:https://doi.org/10.2514/3.46087.

[29] Andrews, S. A., Perez, R. E., and Allan, W. D., "Aerodynamic implications of gull's drooped wing-tips," Bioinspiration and Biomimetics, Vol. 8, No. 4, 2013. doi:https://doi.org/110.1088/1748-3182/8/4/046003.

[30] Nickel, K., and Wohlfahrt, M., Tailless Aircraft in Theory and Practice, AIAA education series, American Institute of Aeronautics and Astronautics, 1994.

[31] Streit, T., Visser, K., and Liersch, C., "Minimal induced drag for non-planar lifting surfaces with moderate and small aspect ratio," Notes on Numerical Fluid Mechanics and Multidisciplinary Design, Vol. 112, 2010, pp. 545-553. doi: https://doi.org/110.1007/978-3-642-14243-7_67.

[32] Bons, N. P., He, X., Mader, C. A., and Martins, J. R., "Multimodality in aerodynamic wing design optimization," AIAA Journal, Vol. 57, No. 3, 2019, pp. 1004-1018. doi:https://doi.org/10.2514/1.J057294. 